Annals of Mathematics, 153 (2001), 149-189

\title{
Hermitian, symmetric and symplectic random ensembles: PDEs for the distribution of the spectrum
}

\author{
By M. Adler and P. van Moerbeke*
}

\begin{abstract}
Given the Hermitian, symmetric and symplectic ensembles, it is shown that the probability that the spectrum belongs to one or several intervals satisfies a nonlinear PDE. This is done for the three classical ensembles: Gaussian, Laguerre and Jacobi. For the Hermitian ensemble, the PDE (in the boundary points of the intervals) is related to the Toda lattice and the KP equation, whereas for the symmetric and symplectic ensembles the PDE is an inductive equation, related to the so-called Pfaff-KP equation and the Pfaff lattice. The method consists of inserting time-variables in the integral and showing that this integral satisfies integrable lattice equations and Virasoro constraints.
\end{abstract}

\section{Contents}

0. Introduction

0.1. Hermitian, symmetric and symplectic Gaussian ensembles

0.2 . Hermitian, symmetric and symplectic Laguerre ensembles

0.3 . Hermitian, symmetric and symplectic Jacobi ensembles

0.4. ODEs, when $E$ has one boundary point

1. Beta-integrals

1.1. Virasoro constraints for $\beta$-integrals

1.2. Proof: $\beta$-integrals as fixed points of vertex operators

1.3. Examples

2. Matrix integrals and associated integrable systems

2.1. Hermitian matrix integrals and the Toda lattice

2.2. Symmetric/symplectic matrix integrals and the Pfaff lattice

3. Expressing $t$-partials in terms of boundary-partials

3.1. Gaussian and Laguerre ensembles

3.2. Jacobi ensemble

3.3. Evaluating the matrix integrals on the full range

* The support of a National Science Foundation grant \#DMS-98-4-50790 is gratefully acknowledged. The support of a National Science Foundation grant \#DMS-98-4-50790, a Nato, a FNRS and a Francqui Foundation grant is gratefully acknowledged. 
4. Proof of Theorems $0.1,0.2,0.3$

4.1. $\beta=2,1$

4.2. $\beta=4$, using duality

4.3. Reduction to Chazy and Painlevé equations $(\beta=2)$

5. Appendix. Self-similarity proof of the Virasoro constraints (Theorem 1.1)

\section{Introduction}

Consider weights of the form $\rho(z) d z:=e^{-V(z)} d z$ on an interval $F=$ $[A, B] \subseteq \mathbb{R}$, with rational logarithmic derivative and subjected to the following boundary conditions:

$$
-\frac{\rho^{\prime}}{\rho}=V^{\prime}=\frac{g}{f}=\frac{\sum_{0}^{\infty} b_{i} z^{i}}{\sum_{0}^{\infty} a_{i} z^{i}}, \quad \lim _{z \rightarrow A, B} f(z) \rho(z) z^{k}=0 \text { for all } k \geq 0,
$$

together with a disjoint union of intervals,

$$
E=\bigcup_{1}^{r}\left[c_{2 i-1}, c_{2 i}\right] \subseteq F \subseteq \mathbb{R}
$$

The data (0.0.1) and (0.0.2) define an algebra of differential operators

$$
\mathcal{B}_{k}=\sum_{1}^{2 r} c_{i}^{k+1} f\left(c_{i}\right) \frac{\partial}{\partial c_{i}} .
$$

Let $\mathcal{H}_{n}, \mathcal{S}_{n}$ and $\mathcal{T}_{n}$ denote the Hermitian $\left(M=\bar{M}^{\top}\right)$, symmetric $\left(M=M^{\top}\right)$ and "symplectic" ensembles $\left(M=\bar{M}^{\top}, M=J \bar{M} J^{-1}\right)$, respectively. Traditionally, the latter is called the "symplectic ensemble," although the matrices involved are not symplectic! These conditions guarantee the reality of the spectrum of $M$. Then, $\mathcal{H}_{n}(E), \mathcal{S}_{n}(E)$ and $\mathcal{T}_{n}(E)$ denote the subsets of $\mathcal{H}_{n}, \mathcal{S}_{n}$ and $\mathcal{T}_{n}$ with spectrum in the subset $E \subseteq F \subseteq \mathbb{R}$. The aim of this paper is to find PDEs for the probabilities

$$
\begin{aligned}
& P_{n}(E):=P_{n}(\text { all spectral points of } M \in E) \\
& =\frac{\int_{\mathcal{H}_{n}(E), \mathcal{S}_{n}(E) \text { or } \mathcal{T}_{n}(E)} e^{-t r V(M)} d M}{\int_{\mathcal{H}_{n}(F), \mathcal{S}_{n}(F) \text { or } \mathcal{T}_{n}(F)} e^{-\operatorname{tr} V(M)} d M} \\
& =\frac{\int_{E^{n}}\left|\Delta_{n}(z)\right|^{\beta} \prod_{k=1}^{n} e^{-V\left(z_{k}\right)} d z_{k}}{\int_{F^{n}}\left|\Delta_{n}(z)\right|^{\beta} \prod_{k=1}^{n} e^{-V\left(z_{k}\right)} d z_{k}}, \quad \beta=2,1,4 \text { respectively, }
\end{aligned}
$$

for the Gaussian, Laguerre and Jacobi weights. The probabilities involve parameters $\beta, a, b$ (see (0.1.1), (0.2.1) and (0.3.2)) and

$$
\delta_{1,4}^{\beta}:=2\left(\left(\frac{\beta}{2}\right)^{1 / 2}-\left(\frac{\beta}{2}\right)^{-1 / 2}\right)^{2}= \begin{cases}0 & \text { for } \beta=2 \\ 1 & \text { for } \beta=1,4 .\end{cases}
$$


The method used to obtain these PDEs involves inserting time-parameters into the integrals, appearing in (0.0.4) and to notice that the integrals obtained satisfy

- Virasoro constraints: linear PDEs in $t$ and the boundary points of $E$, and

- integrable hierarchies:

\begin{tabular}{l|c|l} 
ensemble & $\beta$ & lattice \\
\hline Hermitian & $\beta=2$ & Toda \\
symmetric & $\beta=1$ & Pfaff \\
symplectic & $\beta=4$ & Pfaff
\end{tabular}.

As a consequence of a duality (explained in Theorem 1.1) between $\beta$-Virasoro generators under the map $\beta \mapsto 4 / \beta$, the PDEs obtained have a remarkable property: the coefficients $Q$ and $Q_{i}$ in the PDEs are functions of the variables $n, \beta, a, b$, and have the invariance property under the map

$$
n \rightarrow-2 n, a \rightarrow-\frac{a}{2}, b \rightarrow-\frac{b}{2}
$$

to be precise,

$$
\left.Q_{i}\left(-2 n, \beta,-\frac{a}{2},-\frac{b}{2}\right)\right|_{\beta=1}=\left.Q_{i}(n, \beta, a, b)\right|_{\beta=4} .
$$

Important remark. For $\beta=2$, the probabilities satisfy PDEs in the boundary points of $E$, whereas in the case $\beta=1,4$, the equations are inductive. Namely, for $\beta=1$ (resp. $\beta=4$ ), the probabilities $P_{n+2}$ (resp. $P_{n+1}$ ) are given in terms of $P_{n-2}$ (resp. $\left.P_{n-1}\right)$ and a differential operator acting on $P_{n}$.

0.1. Hermitian, symmetric and symplectic Gaussian ensembles. Given the disjoint union $E \subset \mathbb{R}$ and the weight $e^{-b z^{2}}$, the differential operators $\mathcal{B}_{k}$ take on the form

$$
\mathcal{B}_{k}=\sum_{1}^{2 r} c_{i}^{k+1} \frac{\partial}{\partial c_{i}}
$$

Also, define the invariant polynomials (in the sense of (0.0.5))

and

$$
Q=12 b^{2} n\left(n+1-\frac{2}{\beta}\right), \quad Q_{2}=4\left(1+\delta_{1,4}^{\beta}\right) b\left(2 n+\delta_{1,4}^{\beta}\left(1-\frac{2}{\beta}\right)\right)
$$

$$
Q_{1}=\left(2-\delta_{1,4}^{\beta}\right) \frac{b^{2}}{\beta}
$$

TheOrem 0.1. The following probabilities for $(\beta=2,1,4)$

$$
P_{n}(E)=\frac{\int_{E^{n}}\left|\Delta_{n}(z)\right|^{\beta} \prod_{k=1}^{n} e^{-b z_{k}^{2}} d z_{k}}{\int_{\mathbb{R}^{n}}\left|\Delta_{n}(z)\right|^{\beta} \prod_{k=1}^{n} e^{-b z_{k}^{2}} d z_{k}},
$$


satisfy the PDE's $\left(F:=F_{n}=\log P_{n}\right)$ :

$$
\begin{aligned}
& \delta_{1,4}^{\beta} Q\left(\frac{P_{n-2}{ }_{1} P_{n+{ }_{1}^{2}}}{P_{n}^{2}}-1\right) \text { with index }\left\{\begin{array}{l}
2 \\
2 \text { when } n \text { is even and } \beta=1 \\
1 \text { when } n \text { is arbitrary and } \beta=4
\end{array}\right. \\
& =\left(\mathcal{B}_{-1}^{4}+\left(Q_{2}+6 \mathcal{B}_{-1}^{2} F\right) \mathcal{B}_{-1}^{2}+4 Q_{1}\left(3 \mathcal{B}_{0}^{2}-4 \mathcal{B}_{-1} \mathcal{B}_{1}+6 \mathcal{B}_{0}\right)\right) F
\end{aligned}
$$

0.2. Hermitian, symmetric and symplectic Laguerre ensembles. Given the disjoint union $E \subset \mathbb{R}^{+}$and the weight $z^{a} e^{-b z}$, the $\mathcal{B}_{k}$ take on the form

$$
\mathcal{B}_{k}=\sum_{1}^{2 r} c_{i}^{k+2} \frac{\partial}{\partial c_{i}}
$$

Also define the polynomials, again respecting the duality (0.0.5),

$$
\begin{aligned}
Q & =\left\{\begin{array}{l}
\frac{3}{4} n(n-1)(n+2 a)(n+2 a+1), \quad \text { for } \beta=1 \\
\frac{3}{2} n(2 n+1)(2 n+a)(2 n+a-1), \quad \text { for } \beta=4
\end{array}\right. \\
Q_{2} & =\left(3 \beta n^{2}-\frac{a^{2}}{\beta}+6 a n+4\left(1-\frac{\beta}{2}\right) a+3\right) \delta_{1,4}^{\beta}+\left(1-a^{2}\right)\left(1-\delta_{1,4}^{\beta}\right), \\
Q_{1} & =\left(\beta n^{2}+2 a n+\left(1-\frac{\beta}{2}\right) a\right), \quad Q_{0}=b\left(2-\delta_{1,4}^{\beta}\right)\left(n+\frac{a}{\beta}\right), \\
Q_{-1} & =\frac{b^{2}}{\beta}\left(2-\delta_{1,4}^{\beta}\right) .
\end{aligned}
$$

THEOREM 0.2. The following probabilities

$$
P_{n}(E)=\frac{\int_{E^{n}}\left|\Delta_{n}(z)\right|^{\beta} \prod_{k=1}^{n} z_{k}^{a} e^{-b z_{k}} d z_{k}}{\int_{\mathbb{R}_{+}^{n}}\left|\Delta_{n}(z)\right|^{\beta} \prod_{k=1}^{n} z_{k}^{a} e^{-b z_{k}} d z_{k}}
$$

satisfy the $\mathrm{PDE}$ : $\left(F:=F_{n}=\log P_{n}\right)$

$$
\begin{aligned}
\delta_{1,4}^{\beta} Q( & \left.\frac{P_{n-2}{ }_{1} P_{n+}{ }_{1}^{2}}{P_{n}^{2}}-1\right) \\
= & \left(\mathcal{B}_{-1}^{4}-2\left(\delta_{1,4}^{\beta}+1\right) \mathcal{B}_{-1}^{3}\right. \\
& \quad+\left(Q_{2}+6 \mathcal{B}_{-1}^{2} F-4\left(\delta_{1,4}^{\beta}+1\right) \mathcal{B}_{-1} F\right) \mathcal{B}_{-1}^{2}-3 \delta_{1,4}^{\beta}\left(Q_{1}-\mathcal{B}_{-1} F\right) \mathcal{B}_{-1} \\
& \left.\quad+Q_{-1}\left(3 \mathcal{B}_{0}^{2}-4 \mathcal{B}_{1} \mathcal{B}_{-1}-2 \mathcal{B}_{1}\right)+Q_{0}\left(2 \mathcal{B}_{0} \mathcal{B}_{-1}-\mathcal{B}_{0}\right)\right) F
\end{aligned}
$$

\footnotetext{
${ }^{1}$ with the same convention on the indices $n \pm 2$ and $n \pm 1$, as in (0.1.2)
} 
0.3. Hermitian, symmetric and symplectic Jacobi ensembles. In terms of $E \subset[-1,1]$ and the Jacobi weight $(1-z)^{a}(1+z)^{b}$, the differential operators $\mathcal{B}_{k}$ take on the form

$$
\mathcal{B}_{k}=\sum_{1}^{2 r} c_{i}^{k+1}\left(1-c_{i}^{2}\right) \frac{\partial}{\partial c_{i}}
$$

Setting $b_{0}=a-b, b_{1}=a+b$, we introduce the new variables, which themselves have the invariance property (0.0.5):

$$
\begin{gathered}
r=\frac{4}{\beta}\left(b_{0}^{2}+\left(b_{1}+2-\beta\right)^{2}\right) \quad s=\frac{4}{\beta} b_{0}\left(b_{1}+2-\beta\right) \\
q_{n}=\frac{4}{\beta}\left(\beta n+b_{1}+2-\beta\right)\left(\beta n+b_{1}\right),
\end{gathered}
$$

and the following polynomials in $q=q_{n}, r, s$, thus invariant under the map $(0.0 .5)$ :

$$
\begin{aligned}
Q & =\frac{3}{16}\left(\left(s^{2}-q r+q^{2}\right)^{2}-4\left(r s^{2}-4 q s^{2}-4 s^{2}+q^{2} r\right)\right), \\
Q_{1} & =3 s^{2}-3 q r-6 r+2 q^{2}+23 q+24 \\
Q_{2} & =3 q s^{2}+9 s^{2}-4 q^{2} r+2 q r+4 q^{3}+10 q^{2}, \\
Q_{3} & =3 q s^{2}+6 s^{2}-3 q^{2} r+q^{3}+4 q^{2}, \\
Q_{4} & =9 s^{2}-3 q r-6 r+q^{2}+22 q+24=Q_{1}+\left(6 s^{2}-q^{2}-q\right) .
\end{aligned}
$$

THEOREM 0.3. The following probabilities

$$
P_{n}(E)=\frac{\int_{E^{n}}\left|\Delta_{n}(z)\right|^{\beta} \prod_{k=1}^{n}\left(1-z_{k}\right)^{a}\left(1+z_{k}\right)^{b} d z_{k}}{\int_{[-1,1]^{n}}\left|\Delta_{n}(z)\right|^{\beta} \prod_{k=1}^{n}\left(1-z_{k}\right)^{a}\left(1+z_{k}\right)^{b} d z_{k}}
$$

satisfy the $\operatorname{PDE}\left(F=F_{n}=\log P_{n}\right)$ :

for $\beta=2$ :

$$
\begin{gathered}
\left(2 \mathcal{B}_{-1}^{4}+(q-r+4) \mathcal{B}_{-1}^{2}-\left(4 \mathcal{B}_{-1} F-s\right) \mathcal{B}_{-1}+3 q \mathcal{B}_{0}^{2}-2 q \mathcal{B}_{0}+8 \mathcal{B}_{0} \mathcal{B}_{-1}^{2}\right. \\
\left.-4(q-1) \mathcal{B}_{1} \mathcal{B}_{-1}+\left(4 \mathcal{B}_{-1} F-s\right) \mathcal{B}_{1}+2\left(4 \mathcal{B}_{-1} F-s\right) \mathcal{B}_{0} \mathcal{B}_{-1}+2 q \mathcal{B}_{2}\right) F \\
\quad+4 \mathcal{B}_{-1}^{2} F\left(2 \mathcal{B}_{0} F+3 \mathcal{B}_{-1}^{2} F\right)=0
\end{gathered}
$$


for $\beta=1,4$ :

$$
\begin{aligned}
Q( & \left.\frac{P_{n+{ }_{1}^{2}} P_{n-1}^{2}}{P_{n}^{2}}-1\right) \\
\quad & (q+1)\left(4 q \mathcal{B}_{-1}^{4}+12\left(4 \mathcal{B}_{-1} F-s\right) \mathcal{B}_{-1}^{3}+2(q+12)\left(4 \mathcal{B}_{-1} F-s\right) \mathcal{B}_{0} \mathcal{B}_{-1}\right. \\
& \left.+3 q^{2} \mathcal{B}_{0}^{2}-4(q-4) q \mathcal{B}_{1} \mathcal{B}_{-1}+q\left(4 \mathcal{B}_{-1} F-s\right) \mathcal{B}_{1}+20 q \mathcal{B}_{0} \mathcal{B}_{-1}^{2}+2 q^{2} \mathcal{B}_{2}\right) F \\
& +\left(Q_{2} \mathcal{B}_{-1}^{2}-s Q_{1} \mathcal{B}_{-1}+Q_{3} \mathcal{B}_{0}\right) F+48\left(\mathcal{B}_{-1} F\right)^{4}-48 s\left(\mathcal{B}_{-1} F\right)^{3}+2 Q_{4}\left(\mathcal{B}_{-1} F\right)^{2} \\
& +12 q^{2}\left(\mathcal{B}_{0} F\right)^{2}+16 q(2 q-1)\left(\mathcal{B}_{-1}^{2} F\right)\left(\mathcal{B}_{0} F\right)+24(q-1) q\left(\mathcal{B}_{-1}^{2} F\right)^{2} \\
& +24\left(2 \mathcal{B}_{-1} F-s\right)\left((q+2) \mathcal{B}_{0} F+(q+3) \mathcal{B}_{-1}^{2} F\right) \mathcal{B}_{-1} F
\end{aligned}
$$

0.4. ODEs, when $E$ has one boundary point. Assume the set $E$ consists of one boundary point $c=x$, besides the boundary of the full range. In that case the PDEs in the previous section lead to ODEs in $x$ :

(1) Gaussian $(n \times n)$ matrix ensemble (for the function $\beta=2,1,4)$ :

$$
f_{n}(x)=\frac{d}{d x} \log P_{n}\left(\max _{i} \lambda_{i} \leq x\right)
$$

satisfies

$$
\begin{aligned}
\delta_{1,4}^{\beta} Q & \left(\frac{P_{n-{ }_{1}^{2} P_{n+{ }_{1}^{2}}}}{P_{n}^{2}}-1\right) \\
& =f_{n}^{\prime \prime \prime}+6 f_{n}^{\prime 2}+\left(4 \frac{b^{2} x^{2}}{\beta}\left(\delta_{1,4}^{\beta}-2\right)+Q_{2}\right) f_{n}^{\prime}-4 \frac{b^{2} x}{\beta}\left(\delta_{1,4}^{\beta}-2\right) f_{n} .
\end{aligned}
$$

(2) Laguerre ensemble (for $\beta=2,1,4$ ): all eigenvalues $\lambda_{i}$ satisfy $\lambda_{i} \geq 0$ and

$$
f_{n}(x)=x \frac{d}{d x} \log P_{n}\left(\max _{i} \lambda_{i} \leq x\right)
$$

satisfies (with $f:=f_{n}(x)$ )

$$
\begin{aligned}
\delta_{1,4}^{\beta} Q( & \left.\frac{P_{n-2}{ }_{1} P_{n+{ }_{1}^{2}}}{P_{n}^{2}}-1\right)-\left(3 \delta_{1,4}^{\beta} f-\frac{b^{2} x^{2}}{\beta}\left(\delta_{1,4}^{\beta}-2\right)-Q_{0} x-3 \delta_{1,4}^{\beta} Q_{1}\right) f \\
= & x^{3} f^{\prime \prime \prime}-\left(2 \delta_{1,4}^{\beta}-1\right) x^{2} f^{\prime \prime}+6 x^{2} f^{\prime 2} \\
& \quad-x\left(4\left(\delta_{1,4}^{\beta}+1\right) f-\frac{b^{2} x^{2}}{\beta}\left(\delta_{1,4}^{\beta}-2\right)-2 Q_{0} x-Q_{2}+2 \delta_{1,4}^{\beta}+1\right) f^{\prime} .
\end{aligned}
$$


(3) Jacobi ensemble: all eigenvalues $\lambda_{i}$ satisfy $-1 \leq \lambda_{i} \leq 1$ and

$$
f_{n}(x)=\left(1-x^{2}\right) \frac{d}{d x} \log P_{n}\left(\max _{i} \lambda_{i} \leq x\right)
$$

satisfies (with $f:=f_{n}(x)$ ):

for $\beta=2$ :

$$
\begin{aligned}
& 2\left(x^{2}-1\right)^{2} f^{\prime \prime \prime}+4\left(x^{2}-1\right)\left(x f^{\prime \prime}-3 f^{\prime 2}\right) \\
& \quad+\left(16 x f-q\left(x^{2}-1\right)-2 s x-r\right) f^{\prime}-f(4 f-q x-s)=0
\end{aligned}
$$

for $\beta=1,4$ :

$$
\begin{aligned}
& \text { 4.4) } Q\left(\frac{P_{n+1}^{2} P_{n-1}^{2}}{P_{n}^{2}}-1\right) \\
& =4(q+1)\left(x^{2}-1\right)^{2}\left(-q\left(x^{2}-1\right) f^{\prime \prime \prime}+(12 f-q x-3 s) f^{\prime \prime}+6 q(q-1) f^{\prime 2}\right) \\
& -\left(x^{2}-1\right) f^{\prime}\left(24 f(q+3)(2 f-s)+8 f q(5 q-1) x-q(q+1)\left(q x^{2}+2 s x+8\right)+Q_{2}\right) \\
& +f\left(48 f^{3}+48 f^{2}(q x+2 x-s)+2 f\left(8 q^{2} x^{2}+2 q x^{2}-12 q s x-24 s x+Q_{4}\right)\right. \\
& \left.\quad-q(q+1) x\left(3 q x^{2}+s x-2 q x-3 q\right)+Q_{3} x-Q_{1} s\right) .
\end{aligned}
$$

For $\beta=2, f_{n}(x)$ satisfies a third-order equation (of the so-called Chazytype) with quadratic nonlinearity in $f_{n}^{\prime}$. Then $f_{n}$ also satisfies an equation, which is second-order in $f$ and quadratic in $f^{\prime \prime}$, which after some rescaling can be put in a canonical form. Namely,

$$
\begin{array}{ll}
\text { Gauss } & g_{n}(z)=b^{-1 / 2} f_{n}\left(z b^{-1 / 2}\right)+\frac{2}{3} n z, \\
\text { Laguerre, } & g_{n}(z)=f_{n}(z)+\frac{b}{4}(2 n+a) z+\frac{a^{2}}{4}, \\
\text { Jacobi } & g_{n}(z):=-\left.\frac{1}{2} f_{n}(x)\right|_{x=2 z-1}-\frac{q}{8} z+\frac{q+s}{16}
\end{array}
$$

satisfies the respective canonical equations of Cosgrove [11] and CosgroveScoufis [12],

- $g^{\prime \prime 2}=-4 g^{\prime 3}+4\left(z g^{\prime}-g\right)^{2}+A_{1} g^{\prime}+A_{2}$

(Painlevé IV)

- $\left(z g^{\prime \prime}\right)^{2}=\left(z g^{\prime}-g\right)\left(-4 g^{\prime 2}+A_{1}\left(z g^{\prime}-g\right)+A_{2}\right)+A_{3} g^{\prime}+A_{4}, \quad$ (Painlevé V) 
- $\left(z(z-1) g^{\prime \prime}\right)^{2}=\left(z g^{\prime}-g\right)\left(4 g^{2}-4 g^{\prime}\left(z g^{\prime}-g\right)+A_{2}\right)$

$$
+A_{1} g^{\prime 2}+A_{3} g^{\prime}+A_{4},
$$

(Painlevé VI)

with coefficients which will be determined in Section 4.3. Each of these equations can be transformed into the standard Painlevé equations.

For $\beta=1$ and 4 , the inductive partial differential equations (0.1.2), (0.2.2) and (0.3.4) are new. For $\beta=2$ and for general $E$, they were first computed by Adler-Shiota-van Moerbeke [7], using the method of the present paper. For $\beta=2$ and for $E$ having one boundary point, the equations obtained here coincide with the ones first obtained by Tracy-Widom in [20], who saw them to be Painlevé IV and V for the Gaussian and Laguerre distribution respectively. In his Louvain doctoral dissertation, J. P. Semengue, together with L. Haine [14], were led to Painlevé VI for the Jacobi ensemble, for $\beta=2$ and $E$ having one boundary point, upon subtracting the Tracy-Widom differential equation ([20]) from the ones computed with the Adler-Shiota-van Moerbeke method ([7]). As we shall see, the classification of Cosgrove [11] and Cosgrove-Scoufis [12], (A.3) leads directly to these results.

\section{Beta-integrals}

1.1. Virasoro constraints for $\beta$-integrals. Consider the data from (0.0.1) to (0.0.3) and the $t$-deformations of the integrals (0.0.4), for general $\beta>0$ : $\left(t:=\left(t_{1}, t_{2}, \ldots\right)\right.$ and $\left.c=\left(c_{1}, c_{2}, \ldots, c_{2 r}\right)\right)$

$$
I_{n}(t, c ; \beta):=\int_{E^{n}}\left|\Delta_{n}(z)\right|^{\beta} \prod_{k=1}^{n}\left(e^{\sum_{1}^{\infty} t_{i} z_{k}^{i}} \rho\left(z_{k}\right) d z_{k}\right) \text { for } n>0 .
$$

The main statement of this section is Theorem 1.1, whose proof will be outlined in the next subsection. In Section 5 (Appendix), we give a less conceptual proof, which is based on the invariance of the integral (1.1.2) below, under the

transformation $z_{i} \mapsto z_{i}+\varepsilon f\left(z_{i}\right) z_{i}^{k+1}$ of the integration variables. The central charge (1.1.6) has already appeared in the work of Awata et al. [10].

Theorem 1.1 (Adler-van Moerbeke [2]). The multiple integrals

$$
I_{n}(t, c ; \beta):=\int_{E^{n}}\left|\Delta_{n}(z)\right|^{\beta} \prod_{k=1}^{n}\left(e^{\sum_{1}^{\infty} t_{i} z_{k}^{i}} \rho\left(z_{k}\right) d z_{k}\right) \text { for } n>0
$$

and

$$
I_{n}\left(t, c ; \frac{4}{\beta}\right):=\int_{E^{n}}\left|\Delta_{n}(z)\right|^{4 / \beta} \prod_{k=1}^{n}\left(e^{\sum_{1}^{\infty} t_{i} z_{k}^{i}} \rho\left(z_{k}\right) d z_{k}\right), \text { for } n>0
$$


with $I_{0}=1$, satisfy respectively the following Virasoro constraints 2 for all $k \geq-1$ :

$$
\begin{gathered}
\left(-\mathcal{B}_{k}+\sum_{i \geq 0}\left(a_{i}{ }^{\beta} \mathbb{J}_{k+i, n}^{(2)}(t, n)-b_{i}{ }^{\beta} \mathbb{J}_{k+i+1, n}^{(1)}(t, n)\right)\right) I_{n}(t, c ; \beta)=0, \\
\left(-\mathcal{B}_{k}+\sum_{i \geq 0}\left(a_{i}{ }^{\beta} \mathbb{J}_{k+i, n}^{(2)}\left(-\frac{\beta t}{2},-\frac{2 n}{\beta}\right)\right.\right. \\
\left.\left.+\frac{\beta b_{i}}{2}{ }^{\beta} \mathbb{J}_{k+i+1, n}^{(1)}\left(-\frac{\beta t}{2},-\frac{2 n}{\beta}\right)\right)\right) I_{n}\left(t, c ; \frac{4}{\beta}\right)=0,
\end{gathered}
$$

in terms of the coefficients $a_{i}, b_{i}$ of the rational function $(-\log \rho)^{\prime}$ and the end points $c_{i}$ of the subset $E$, as in (0.0.1) to (0.0.3). For all $n \in \mathbb{Z}$, the ${ }^{\beta} \mathbb{J}_{k, n}^{(2)}(t, n)$ and $\beta \mathbb{J}_{k, n}^{(1)}(t, n)$ form a Virasoro and a Heisenberg algebra respectively, interacting as follows:

$$
\begin{aligned}
{\left[{ }^{\beta} \mathbb{J}_{k, n}^{(2)},{ }^{\beta} \mathbb{J}_{\ell, n}^{(2)}\right] } & =(k-\ell)^{\beta} \mathbb{J}_{k+\ell, n}^{(2)}+c\left(\frac{k^{3}-k}{12}\right) \delta_{k,-\ell} \\
{\left[{ }^{\beta} \mathbb{J}_{k, n}^{(2)},{ }^{\beta} \mathbb{J}_{\ell, n}^{(1)}\right] } & =-\ell{ }^{\beta} \mathbb{J}_{k+\ell, n}^{(1)}+c^{\prime} k(k+1) \delta_{k,-\ell} \\
{\left[{ }^{\beta} \mathbb{J}_{k, n}^{(1)},{ }^{\beta} \mathbb{J}_{\ell, n}^{(1)}\right] } & =\frac{k}{\beta} \delta_{k,-\ell},
\end{aligned}
$$

with central charge

$$
c=1-6\left(\left(\frac{\beta}{2}\right)^{1 / 2}-\left(\frac{\beta}{2}\right)^{-1 / 2}\right)^{2} \quad \text { and } \quad c^{\prime}=\left(\frac{1}{\beta}-\frac{1}{2}\right) .
$$

Remark 1. The ${ }^{\beta} \mathbb{J}_{k, n}^{(2)}$ 's are defined as follows:

$$
{ }^{\beta} \mathbb{J}_{k, n}^{(2)}=\frac{\beta}{2} \sum_{i+j=k}:{ }^{\beta} \mathbb{J}_{i, n}^{(1)} \beta \mathbb{J}_{j, n}^{(1)}:+\left(1-\frac{\beta}{2}\right)\left((k+1)^{\beta} \mathbb{J}_{k, n}^{(1)}-k \mathbb{J}_{k, n}^{(0)}\right) .
$$

Componentwise, we have

$$
{ }^{\beta} \mathbb{J}_{k, n}^{(1)}(t, n)={ }^{\beta} J_{k}^{(1)}+n J_{k}^{(0)} \text { and }{ }^{\beta} \mathbb{J}_{k, n}^{(0)}=n J_{k}^{(0)}=n \delta_{0 k}
$$

and hence

$$
\begin{aligned}
{ }^{\beta} \mathbb{J}_{k, n}^{(2)}(t, n)= & \left(\frac{\beta}{2}\right){ }^{\beta} J_{k}^{(2)}+\left(n \beta+(k+1)\left(1-\frac{\beta}{2}\right)\right){ }^{\beta} J_{k}^{(1)} \\
& +n\left((n-1) \frac{\beta}{2}+1\right) J_{k}^{(0)}
\end{aligned}
$$

\footnotetext{
${ }^{2}$ When $E$ equals the whole range $F$, then the $\mathcal{B}_{k}$ 's are absent in the formulae (1.1.4).
} 
where

$$
\begin{aligned}
& { }^{\beta} J_{k}^{(1)}=\frac{\partial}{\partial t_{k}}+\frac{1}{\beta}(-k) t_{-k} \\
& { }^{\beta} J_{k}^{(2)}=\sum_{i+j=k} \frac{\partial^{2}}{\partial t_{i} \partial t_{j}}+\frac{2}{\beta} \sum_{-i+j=k} i t_{i} \frac{\partial}{\partial t_{j}}+\frac{1}{\beta^{2}} \sum_{-i-j=k} i t_{i} j t_{j} .
\end{aligned}
$$

We put $n$ explicitly in ${ }^{\beta} \mathbb{J}_{\ell, n}^{(2)}(t, n)$ to indicate that the $n^{\text {th }}$ component contains $n$ explicitly, besides $t$.

Remark 2. The Heisenberg and Virasoro generators satisfy the following duality properties:

$$
\begin{aligned}
& \frac{4}{\beta} \mathbb{J}_{\ell, n}^{(2)}(t, n)=\beta \mathbb{J}_{\ell, n}^{(2)}\left(-\frac{\beta t}{2},-\frac{2 n}{\beta}\right), n \in \mathbb{Z} \\
& \frac{4}{\beta} \mathbb{J}_{\ell, n}^{(1)}(t, n)=-\frac{\beta}{2} \beta \mathbb{J}_{\ell, n}^{(1)}\left(-\frac{\beta t}{2},-\frac{2 n}{\beta}\right), n>0 .
\end{aligned}
$$

In (1.1.9), ${ }^{\beta} \mathbb{J}_{\ell, n}^{(2)}(-\beta t / 2,-2 n / \beta)$ means that the variable $n$, which appears in the $n^{\text {th }}$ component, gets replaced by $-2 n / \beta$ and $t$ by $-\beta t / 2$.

1.2. Proof: $\beta$-integrals as fixed points of vertex operators. The most transparent way to prove Theorem 1.1 is via vector vertex operators, for which the $\beta$-integrals are fixed points. This is a technique which has been used by us already in [1]. Indeed, define the (vector) vertex operator $\mathbb{X}$, for $t=\left(t_{1}, t_{2}, \ldots\right) \in \mathbb{C}^{\infty}, u \in \mathbb{C}:$

$$
\mathbb{X}_{\beta}(t, u)=\Lambda^{-1} e^{\sum_{1}^{\infty} t_{i} u^{i}} e^{-\beta \sum_{1}^{\infty} \frac{u^{-i}}{i} \frac{\partial}{\partial t_{i}}} \chi\left(|u|^{\beta}\right),
$$

where $\chi(z):=\left(1, z, z^{2}, \ldots\right)$. The vertex operator acts on vectors $f(t)=$ $\left(f_{0}(t), f_{1}(t), \ldots\right)$ of functions, as follows

$$
\left(\mathbb{X}_{\beta}(t, u) f(t)\right)_{n}=e^{\sum_{1}^{\infty} t_{i} u^{i}}\left(|u|^{\beta}\right)^{n-1} f_{n-1}\left(t-\beta\left[u^{-1}\right]\right) .
$$

For the sake of convenience, in this section we introduce the following vector Virasoro generators: ${ }^{\beta} \mathbb{J}_{k}^{(i)}(t):=\left({ }^{\beta} \mathbb{J}_{k, n}^{(i)}(t, n)\right)_{n \in \mathbb{Z}}$.

Proposition 1.2. The multiplication operator $z^{k}$ and the differential operators $\frac{\partial}{\partial z} z^{k+1}$ with $z \in \mathbb{C}^{*}$, acting on the vertex operator $\mathbb{X}_{\beta}(t, z)$, have realizations as commutators, in terms of the Heisenberg and Virasoro generators

\footnotetext{
${ }^{3}$ For $\alpha \in \mathbb{C}$, define $[\alpha]:=\left(\alpha, \frac{\alpha^{2}}{2}, \frac{\alpha^{3}}{3}, \ldots\right) \in \mathbb{C}^{\infty}$. The operator $\Lambda$ is the shift matrix, with zeroes
} everywhere, except for 1 's just above the diagonal, i.e., $(\Lambda v)_{n}=v_{n+1}$. 
${ }^{\beta} \mathbb{J}_{k}^{(1)}(t)$ and ${ }^{\beta} \mathbb{J}_{k}^{(2)}(t):$

$$
\begin{aligned}
z^{k} \mathbb{X}_{\beta}(t, z) & =\left[{ }^{\beta} \mathbb{J}_{k}^{(1)}(t), \mathbb{X}_{\beta}(t, z)\right], \\
\frac{\partial}{\partial z} z^{k+1} \mathbb{X}_{\beta}(t, z) & =\left[{ }^{\beta} \mathbb{J}_{k}^{(2)}(t), \mathbb{X}_{\beta}(t, z)\right] .
\end{aligned}
$$

Corollary 1.3. Given a weight $\rho(z) d z$ on $\mathbb{R}$ satisfying (0.0.1), we have $(1.2 .3)$

$$
\frac{\partial}{\partial z} z^{k+1} f(z) \mathbb{X}_{\beta}(t, z) \rho(z)=\left[\sum_{i \geq 0}\left(a_{i}{ }^{\beta} \mathbb{J}_{k+i}^{(2)}(t)-b_{i}{ }^{\beta} \mathbb{J}_{k+i+1}^{(1)}(t)\right), \mathbb{X}_{\beta}(t, z) \rho(z)\right]
$$

Proof. Using (1.2.2) in the last line, compute

$$
\begin{aligned}
& \frac{\partial}{\partial z} z^{k+1} f(z) \mathbb{X}_{\beta}(t, z) \rho(z) \\
& \quad=\left(\frac{\rho^{\prime}(z)}{\rho(z)} f(z)\right) z^{k+1} \mathbb{X}_{\beta}(t, z) \rho(z)+\rho(z) \frac{\partial}{\partial z}\left(z^{k+1} f(z) \mathbb{X}_{\beta}(t, z)\right) \\
& \quad=-\left(\sum_{0}^{\infty} b_{i} z^{k+i+1} \mathbb{X}_{\beta}(t, z)\right) \rho(z)+\rho(z) \frac{\partial}{\partial z}\left(\sum_{0}^{\infty} a_{i} z^{k+i+1} \mathbb{X}_{\beta}(t, z)\right) \\
& \quad=-\left[\sum_{0}^{\infty} b_{i}{ }^{\beta} \mathbb{J}_{k+i+1}^{(1)}, \mathbb{X}_{\beta}(t, z) \rho(z)\right]+\left[\sum_{0}^{\infty} a_{i}{ }^{\beta} \mathbb{J}_{k+i}^{(2)}, \mathbb{X}_{\beta}(t, z) \rho(z)\right]
\end{aligned}
$$

establishing (1.2.3).

Given the weight $\rho_{E}(u) d u=\rho(u) I_{E}(u) d u$, with $\rho$ and $E$ as before, and with $I_{E}$ the indicator function of $E$, define the integrated vector vertex operator

$$
\mathbb{Y}_{\beta}\left(t, \rho_{E}\right):=\int_{E} d u \rho(u) \mathbb{X}_{\beta}(t, u),
$$

and the vector operator

$$
\begin{aligned}
\mathcal{D}_{k} & :=\mathcal{B}_{k}-\mathcal{V}_{k} \\
& :=\sum_{1}^{2 r} c_{i}^{k+1} f\left(c_{i}\right) \frac{\partial}{\partial c_{i}}-\sum_{i \geq 0}\left(a_{i}{ }^{\beta} \mathbb{J}_{k+i}^{(2)}(t)-b_{i}{ }^{\beta} \mathbb{J}_{k+i+1}^{(1)}(t)\right)
\end{aligned}
$$

consisting of a $c$-dependent boundary part $\mathcal{B}_{k}$ and a $(t, n)$-dependent Virasoro part $\mathcal{V}_{k}$.

Proposition 1.4. The following commutation relation holds:

$$
\left[\mathcal{D}_{k}, \mathbb{Y}_{\beta}\left(t, \rho_{E}\right)\right]=0 \text {. }
$$


Proof. Integrating both sides of (1.2.3) over $E$, one computes:

$$
\begin{aligned}
\int_{E} d z \frac{\partial}{\partial z}\left(z^{k+1} f(z) \mathbb{X}_{\beta}(t, z) \rho(z)\right) & =\sum_{1}^{2 r}(-1)^{i} c_{i}^{k+1} f\left(c_{i}\right) \mathbb{X}_{\beta}\left(t, c_{i}\right) \rho\left(c_{i}\right) \\
& =\sum_{1}^{2 r} c_{i}^{k+1} f\left(c_{i}\right) \frac{\partial}{\partial c_{i}} \int_{E} \mathbb{X}_{\beta}(t, z) \rho(z) d z \\
& =\left[\mathcal{B}_{k}, \mathbb{Y}_{\beta}\left(t, \rho_{E}\right)\right]
\end{aligned}
$$

while on the other hand

$$
\begin{aligned}
\int_{E} d z & {\left[\sum_{i \geq 0}\left(a_{i}{ }^{\beta} \mathbb{J}_{k+i}^{(2)}-b_{i}{ }^{\beta} \mathbb{J}_{k+i+1}^{(1)}\right), \mathbb{X}_{\beta}(t, z) \rho(z)\right] } \\
& =\left[\sum_{i \geq 0}\left(a_{i}{ }^{\beta} \mathbb{J}_{k+i}^{(2)}-b_{i}{ }^{\beta} \mathbb{J}_{k+i+1}^{(1)}\right), \int_{E} d z \rho(z) \mathbb{X}_{\beta}(t, z)\right] \\
& =\left[\mathcal{V}_{k}, \mathbb{Y}_{\beta}\left(t, \rho_{E}\right)\right] .
\end{aligned}
$$

Subtracting both expressions (1.2.8) and (1.2.9) yields, using (1.2.3),

$$
0=\left[\mathcal{B}_{k}-\mathcal{V}_{k}, \mathbb{Y}_{\beta}\left(t, \rho_{E}\right)\right]=\left[\mathcal{D}_{k}, \mathbb{Y}_{\beta}\left(t, \rho_{E}\right)\right],
$$

concluding the proof of Proposition 1.4.

Proposition 1.5. The column vector,

$$
I(t):=\left(\int_{E^{n}}\left|\Delta_{n}(z)\right|^{\beta} \prod_{k=1}^{n} e^{\sum_{1}^{\infty} t_{i} z_{k}^{i}} \rho\left(z_{k}\right) d z_{k}\right)_{n \geq 0}
$$

is a fixed point for the vertex operator $\mathbb{Y}_{\beta}\left(t, \rho_{E}\right)$ :

$$
\left(\mathbb{Y}_{\beta}\left(t, \rho_{E}\right) I\right)_{n}=I_{n}, n \geq 1 \text {. }
$$

Proof. We have

$$
\begin{aligned}
I_{n}(t)= & \int_{\mathbb{R}^{n}}\left|\Delta_{n}(z)\right|^{\beta} \prod_{k=1}^{n}\left(e^{\sum_{1}^{\infty} t_{i} z_{k}^{i}} \rho_{E}\left(z_{k}\right) d z_{k}\right) \\
= & \int_{\mathbb{R}} d u \rho_{E}(u) e^{\sum_{1}^{\infty} t_{i} u^{i}}|u|^{\beta(n-1)} \\
& \quad \int_{\mathbb{R}^{n-1}} \prod_{k=1}^{n-1}\left|1-\frac{z_{k}}{u}\right|^{\beta}\left|\Delta_{n-1}(z)\right|^{\beta} \prod_{k=1}^{n-1}\left(e^{\sum_{1}^{\infty} t_{i} z_{k}^{i}} \rho_{E}\left(z_{k}\right) d z_{k}\right)
\end{aligned}
$$




$$
\begin{aligned}
& =\int_{\mathbb{R}} d u \rho_{E}(u) e^{\sum_{1}^{\infty} t_{i} u^{i}}|u|^{\beta(n-1)} \\
& \quad e^{-\beta \sum_{1}^{\infty} \frac{u^{-i}}{i} \frac{\partial}{\partial t_{i}}} \int_{\mathbb{R}^{n-1}}\left|\Delta_{n-1}(z)\right|^{\beta} \prod_{k=1}^{n-1}\left(e^{\sum_{1}^{\infty} t_{i} z_{k}^{i}} \rho_{E}\left(z_{k}\right) d z_{k}\right) \\
& =\int_{\mathbb{R}} d u \rho_{E}(u)|u|^{\beta(n-1)} e^{\sum_{1}^{\infty} t_{i} u^{i}} e^{-\beta \sum_{1}^{\infty} \frac{u^{-i}}{i} \frac{\partial}{\partial t_{i}}} I_{n-1}(t) \\
& =\left(\mathbb{Y}_{\beta}\left(t, \rho_{E}\right) I(t)\right)_{n} .
\end{aligned}
$$

It suffices to do the above argument for all $t_{i}>0$, enabling one to replace $e^{\sum_{1}^{\infty} t_{i} z^{i}}$ by $\left|e^{\sum_{1}^{\infty} t_{i} z^{i}}\right|$. Then one continues the result for all $t_{i} \in \mathbb{C}$.

Proof of Theorem 1.1. From Proposition 1.4 it follows that for $n \geq 1$,

$$
\begin{aligned}
0 & =\left[\mathcal{D}_{k},\left(\mathbb{Y}_{\beta}\left(t, \rho_{E}\right)\right)^{n}\right] I \\
& =\mathcal{D}_{k} \mathbb{Y}_{\beta}\left(t, \rho_{E}\right)^{n} I-\mathbb{Y}_{\beta}\left(t, \rho_{E}\right)^{n} \mathcal{D}_{k} I .
\end{aligned}
$$

Taking the $n^{\text {th }}$ component for $n \geq 1$ and $k \geq-1$, setting

$$
X_{\beta}(t, u)=e^{\sum t_{i} u^{i}} e^{-\beta \sum \frac{u^{-i}}{i} \frac{\partial}{\partial t_{i}}},
$$

and using (1.2.10), we have

$$
\begin{aligned}
0 & =\left(\mathcal{D}_{k} I-\mathbb{Y}_{\beta}\left(t, \rho_{E}\right)^{n} \mathcal{D}_{k} I\right)_{n} \\
& =\left(\mathcal{D}_{k} I\right)_{n}-\int d u \rho_{E}(u) X_{\beta}(t ; u)\left(|u|^{\beta}\right)^{n-1} \ldots \int d u \rho_{E}(u) X_{\beta}(t ; u)\left(\mathcal{D}_{k} I\right)_{0} \\
& =\left(\mathcal{D}_{k} I\right)_{n} .
\end{aligned}
$$

Indeed $\left(\mathcal{D}_{k} I\right)_{0}=0$ for $k \geq-1$, since $I_{0}=1$ and $\mathcal{D}_{k}$ involves $\mathcal{B}_{k},{ }^{\beta} J_{k}^{(2)},{ }^{\beta} J_{k}^{(1)}$ and $J_{k}^{(0)}$ for $k \geq-1$ :

$$
\left\{\begin{array}{l}
\mathcal{B}_{k} \text { and }{ }^{\beta} J_{k}^{(2)} \text { are pure differentiations for } k \geq-1 ; \\
{ }^{\beta} J_{k}^{(1)} \text { is pure differentiation, except for } k=-1 ; \\
{ }^{\beta} J_{-1}^{(1)} \text { appears with coefficient } n \beta \text {, which vanishes for } n=0 ; \\
J_{k}^{(0)} \text { appears with coefficient } n\left((n-1) \frac{\beta}{2}+1\right) \text {, vanishing for } n=0 .
\end{array}\right.
$$

The proof of the $2^{\text {nd }}$ formula in (1.1.4) follows immediately from the duality (1.1.9).

1.3. Examples. Example 1 (Gaussian $\beta$-integrals). The weight and the $a_{i}$ and $b_{i}$, as in (0.0.1), are given by (setting $b=1$ in (0.1.1))

$$
\begin{gathered}
\rho(z)=e^{-V(z)}=e^{-z^{2}}, \quad V^{\prime}=g / f=2 z, \\
a_{0}=1, b_{0}=0, b_{1}=2, \text { and all other } a_{i}, b_{i}=0 .
\end{gathered}
$$


From Theorem 1.1, the integrals

$$
I_{n}=\int_{E^{n}}\left|\Delta_{n}(z)\right|^{\beta} \prod_{k=1}^{n} e^{-z_{k}^{2}+\sum_{i=1}^{\infty} t_{i} z_{k}^{i}} d z_{k}
$$

satisfy the Virasoro constraints

$$
-\mathcal{B}_{k} I_{n}=-\sum_{1}^{2 r} c_{i}^{k+1} \frac{\partial}{\partial c_{i}} I_{n}=\left(-{ }^{\beta} \mathbb{J}_{k, n}^{(2)}+2^{\beta} \mathbb{J}_{k+2, n}^{(1)}\right) I_{n}, \quad k=-1,0,1, \ldots
$$

Introducing the following notation

$$
\sigma_{i}=\left(n-\frac{i+1}{2}\right) \beta+i+1-b_{0}=\left(n-\frac{i+1}{2}\right) \beta+i+1,
$$

and upon setting $F=\log I_{n}$ we find that the first three constraints have the following form:

$$
\begin{aligned}
-\mathcal{B}_{-1} F & =\left(2 \frac{\partial}{\partial t_{1}}-\sum_{i \geq 2} i t_{i} \frac{\partial}{\partial t_{i-1}}\right) F-n t_{1}, \\
-\mathcal{B}_{0} F & =\left(2 \frac{\partial}{\partial t_{2}}-\sum_{i \geq 1} i t_{i} \frac{\partial}{\partial t_{i}}\right) F-\frac{n}{2} \sigma_{1}, \\
-\mathcal{B}_{1} F & =\left(2 \frac{\partial}{\partial t_{3}}-\sigma_{1} \frac{\partial}{\partial t_{1}}-\sum_{i \geq 1} i t_{i} \frac{\partial}{\partial t_{i+1}}\right) F .
\end{aligned}
$$

For later use, take linear combinations such that each expression contains the pure differentiation term $\partial F / \partial t_{i}$ :

$$
\mathcal{D}_{1}=-\frac{1}{2} \mathcal{B}_{-1}, \quad \mathcal{D}_{2}=-\frac{1}{2} \mathcal{B}_{0}, \quad \mathcal{D}_{3}=-\frac{1}{2}\left(\mathcal{B}_{1}+\frac{\sigma_{1}}{2} \mathcal{B}_{-1}\right),
$$

which yields

$$
\begin{aligned}
& \mathcal{D}_{1} F=\left(\frac{\partial}{\partial t_{1}}-\frac{1}{2} \sum_{i \geq 2} i t_{i} \frac{\partial}{\partial t_{i-1}}\right) F-\frac{n t_{1}}{2} \\
& \mathcal{D}_{2} F=\left(\frac{\partial}{\partial t_{2}}-\frac{1}{2} \sum_{i \geq 1} i t_{i} \frac{\partial}{\partial t_{i}}\right) F-\frac{n}{4} \sigma_{1}, \\
& \mathcal{D}_{3} F=\left(\frac{\partial}{\partial t_{3}}-\frac{1}{2} \sum_{i \geq 1} i t_{i} \frac{\partial}{\partial t_{i+1}}-\frac{1}{4} \sigma_{1} \sum_{i \geq 2} i t_{i} \frac{\partial}{\partial t_{i-1}}\right) F-\frac{n}{4} \sigma_{1} t_{1} .
\end{aligned}
$$


Example 2 (Laguerre $\beta$-integrals). Here, the weight and the $a_{i}$ and $b_{i}$, as in (0.0.1), are given by (again setting $b=1$ in (0.2.1))

$$
\begin{gathered}
e^{-V}=z^{a} e^{-z}, \quad V^{\prime}=\frac{g}{f}=\frac{z-a}{z}, \\
a_{0}=0, a_{1}=1, b_{0}=-a, b_{1}=1, \text { and all other } a_{i}, b_{i}=0 .
\end{gathered}
$$

Thus from (1.1.4), the integrals

$$
I_{n}=\int_{E^{n}}\left|\Delta_{n}(z)\right|^{\beta} \prod_{k=1}^{n} z_{k}^{a} e^{-z_{k}+\sum_{i=1}^{\infty} t_{i} z_{k}^{i}} d z_{k}
$$

satisfy the Virasoro constraints, for $k \geq-1$,

$$
-\mathcal{B}_{k} I_{n}=-\sum_{1}^{2 r} c_{i}^{k+2} \frac{\partial}{\partial c_{i}} I_{n}=\left(-{ }^{\beta} \mathbb{J}_{k+1, n}^{(2)}-a^{\beta} \mathbb{J}_{k+1, n}^{(1)}+{ }^{\beta} \mathbb{J}_{k+2, n}^{(1)}\right) I_{n} .
$$

Introducing the following notation, as before,

$$
\sigma_{i}=\left(n-\frac{i+1}{2}\right) \beta+i+1-b_{0}=\left(n-\frac{i+1}{2}\right) \beta+i+1+a,
$$

and upon setting $F=F_{n}=\log I_{n}$, we see that the first three have the form:

$$
\begin{aligned}
-\mathcal{B}_{-1} F & =\left(\frac{\partial}{\partial t_{1}}-\sum_{i \geq 1} i t_{i} \frac{\partial}{\partial t_{i}}\right) F-\frac{n}{2}\left(\sigma_{1}+a\right), \\
-\mathcal{B}_{0} F & =\left(\frac{\partial}{\partial t_{2}}-\sigma_{1} \frac{\partial}{\partial t_{1}}-\sum_{i \geq 1} i t_{i} \frac{\partial}{\partial t_{i+1}}\right) F \\
-\mathcal{B}_{1} F & =\left(\frac{\partial}{\partial t_{3}}-\sigma_{2} \frac{\partial}{\partial t_{2}}-\sum_{i \geq 1} i t_{i} \frac{\partial}{\partial t_{i+2}}-\frac{\beta}{2} \frac{\partial^{2}}{\partial t_{1}^{2}}\right) F-\frac{\beta}{2}\left(\frac{\partial F}{\partial t_{1}}\right)^{2} .
\end{aligned}
$$

Replacing the operators $\mathcal{B}_{i}$ by linear combinations $\mathcal{D}_{i}$, we see that

$$
\begin{aligned}
& \mathcal{D}_{1}=-\mathcal{B}_{-1} \\
& \mathcal{D}_{2}=-\mathcal{B}_{0}-\sigma_{1} \mathcal{B}_{-1} \\
& \mathcal{D}_{3}=-\mathcal{B}_{1}-\sigma_{2} \mathcal{B}_{0}-\sigma_{1} \sigma_{2} \mathcal{B}_{-1}
\end{aligned}
$$

yields expressions, each containing a pure derivative $\partial F / \partial t_{i}$

$$
\begin{aligned}
& \mathcal{D}_{1} F=\frac{\partial F}{\partial t_{1}}-\sum_{i \geq 1} i t_{i} \frac{\partial F}{\partial t_{i}}-\frac{n}{2}\left(\sigma_{1}+a\right) \\
& \mathcal{D}_{2} F=\frac{\partial F}{\partial t_{2}}+\sum_{i \geq 1} i t_{i}\left(-\sigma_{1} \frac{\partial}{\partial t_{i}}-\frac{\partial}{\partial t_{i+1}}\right) F-\frac{n}{2}\left(\sigma_{1}+a\right) \sigma_{1}
\end{aligned}
$$




$$
\begin{aligned}
\mathcal{D}_{3} F= & \frac{\partial F}{\partial t_{3}}-\sum_{i \geq 1} i t_{i}\left(\sigma_{1} \sigma_{2} \frac{\partial}{\partial t_{i}}+\sigma_{2} \frac{\partial}{\partial t_{i+1}}+\frac{\partial}{\partial t_{i+2}}\right) F-\frac{n}{2}\left(\sigma_{1}+a\right) \sigma_{1} \sigma_{2} \\
& -\frac{\beta}{2}\left(\frac{\partial^{2} F}{\partial t_{1}^{2}}+\left(\frac{\partial F}{\partial t_{1}}\right)^{2}\right)
\end{aligned}
$$

Example 3 (Jacobi $\beta$-integral). The weight and the $a_{i}$ and $b_{i}$, as in (0.0.1), are given by

$$
\begin{gathered}
\rho_{a b}(z):=e^{-V}=(1-z)^{a}(1+z)^{b}, V^{\prime}=\frac{g}{f}=\frac{a-b+(a+b) z}{1-z^{2}}, \\
a_{0}=1, a_{1}=0, a_{2}=-1, b_{0}=a-b, b_{1}=a+b, \text { and all other } a_{i}, b_{i}=0 .
\end{gathered}
$$

The integrals

$$
\int_{E^{n}}\left|\Delta_{n}(z)\right|^{\beta} \prod_{k=1}^{n}\left(1-z_{k}\right)^{a}\left(1+z_{k}\right)^{b} e^{\sum_{i=1}^{\infty} t_{i} z_{k}^{i}} d z_{k}
$$

satisfy the Virasoro constraints $(k \geq-1)$ :

$$
\begin{aligned}
-\mathcal{B}_{k} I_{n} & =-\sum_{1}^{2 r} c_{i}^{k+1}\left(1-c_{i}^{2}\right) \frac{\partial}{\partial c_{i}} I_{n} \\
& =\left({ }^{\beta} \mathbb{J}_{k+2, n}^{(2)}-\beta \mathbb{J}_{k, n}^{(2)}+b_{0}{ }^{\beta} \mathbb{J}_{k+1, n}^{(1)}+b_{1}{ }^{\beta} \mathbb{J}_{k+2, n}^{(1)}\right) I_{n} .
\end{aligned}
$$

Introducing the following notation,

$$
\sigma_{i}=\left(n-\frac{i+1}{2}\right) \beta+i+1+b_{1},
$$

and upon setting $F=F_{n}=\log I_{n}$, we see that the first four have the following form:

$$
\begin{aligned}
-\mathcal{B}_{-1} F= & \left(\sigma_{1} \frac{\partial}{\partial t_{1}}+\sum_{i \geq 1} i t_{i} \frac{\partial}{\partial t_{i+1}}-\sum_{i \geq 2} i t_{i} \frac{\partial}{\partial t_{i-1}}\right) F+n\left(b_{0}-t_{1}\right), \\
-\mathcal{B}_{0} F= & \left(\sigma_{2} \frac{\partial}{\partial t_{2}}+b_{0} \frac{\partial}{\partial t_{1}}+\sum_{i \geq 1} i t_{i}\left(\frac{\partial}{\partial t_{i+2}}-\frac{\partial}{\partial t_{i}}\right)+\frac{\beta}{2} \frac{\partial^{2}}{\partial t_{1}^{2}}\right) F \\
& +\frac{\beta}{2}\left(\frac{\partial F}{\partial t_{1}}\right)^{2}-\frac{n}{2}\left(\sigma_{1}-b_{1}\right), \\
-\mathcal{B}_{1} F= & \left(\sigma_{3} \frac{\partial}{\partial t_{3}}+b_{0} \frac{\partial}{\partial t_{2}}-\left(\sigma_{1}-b_{1}\right) \frac{\partial}{\partial t_{1}}+\sum_{i \geq 1} i t_{i}\left(\frac{\partial}{\partial t_{i+3}}-\frac{\partial}{\partial t_{i+1}}\right)\right. \\
& \left.+\beta \frac{\partial^{2}}{\partial t_{1} \partial t_{2}}\right) F+\beta \frac{\partial F}{\partial t_{1}} \frac{\partial F}{\partial t_{2}},
\end{aligned}
$$




$$
\begin{aligned}
-\mathcal{B}_{2} F= & \left(\sigma_{4} \frac{\partial}{\partial t_{4}}+b_{0} \frac{\partial}{\partial t_{3}}-\left(\sigma_{2}-b_{1}\right) \frac{\partial}{\partial t_{2}}+\sum_{i \geq 1} i t_{i}\left(\frac{\partial}{\partial t_{i+4}}-\frac{\partial}{\partial t_{i+2}}\right)\right. \\
& \left.+\frac{\beta}{2}\left(\frac{\partial^{2}}{\partial t_{2}^{2}}-\frac{\partial^{2}}{\partial t_{1}^{2}}+2 \frac{\partial^{2}}{\partial t_{1} \partial t_{3}}\right)\right) F+\frac{\beta}{2}\left(\left(\frac{\partial F}{\partial t_{2}}\right)^{2}-\left(\frac{\partial F}{\partial t_{1}}\right)^{2}+2 \frac{\partial F}{\partial t_{1}} \frac{\partial F}{\partial t_{3}}\right) .
\end{aligned}
$$

\section{Matrix integrals and associated integrable systems}

2.1. Hermitian matrix integrals and the Toda lattice. Given a weight $\rho(z)=e^{-V(z)}$ defined as in (0.0.1), the inner-product

$$
\langle f, g\rangle_{t}=\int_{E} f(z) g(z) \rho_{t}(z) d z, \quad \text { with } \rho_{t}:=e^{\sum_{1}^{\infty} t_{i} z^{i}} \rho(z),
$$

leads to a moment matrix

$$
m_{n}(t)=\left(\mu_{i j}(t)\right)_{0 \leq i, j<n}=\left(\left\langle z^{i}, z^{j}\right\rangle_{t}\right)_{0 \leq i, j<n},
$$

which is a Hänkel matrix, thus symmetric. Hänkel is tantamount to $\Lambda m_{\infty}=$ $m_{\infty} \Lambda^{\top}$. The semi-infinite moment matrix $m_{\infty}$ evolves in $t$ according to the equations

$$
\frac{\partial \mu_{i j}}{\partial t_{k}}=\mu_{i+k, j}, \text { and thus } \frac{\partial m_{\infty}}{\partial t_{k}}=\Lambda^{k} m_{\infty} \quad\left(\begin{array}{l}
\text { commuting } \\
\text { vector fields }
\end{array}\right) .
$$

Another important ingredient is the factorization of $m_{\infty}$ into a lower- times an upper-triangular matrix

$$
m_{\infty}(t)=S(t)^{-1} S(t)^{\top-1},
$$

where $S(t)$ is lower-triangular with nonzero diagonal elements.

TheOREM 2.1. The vector $\tau(t)=\left(\tau_{n}(t)\right)_{n \geq 0}$, with

$$
\tau_{n}(t):=\operatorname{det} m_{n}(t)=\frac{1}{n !} \int_{E^{n}} \Delta_{n}^{2}(z) \prod_{k=1}^{n} \rho_{t}\left(z_{k}\right) d z_{k}
$$

satisfies:

(i) Virasoro constraints (1.1.4) for $\beta=2$,

$$
\left(-\sum_{1}^{2 r} c_{i}^{k+1} f\left(c_{i}\right) \frac{\partial}{\partial c_{i}}+\sum_{i \geq 0}\left(a_{i} \mathbb{J}_{k+i}^{(2)}-b_{i} \mathbb{J}_{k+i+1}^{(1)}\right)\right) \tau=0
$$

\footnotetext{
${ }^{4}$ Hänkel means $\mu_{i j}$ depends on $i+j$ only.

${ }^{5}$ This factorization is possible for those $t$ 's for which $\tau_{n}(t):=\operatorname{det} m_{n}(t) \neq 0$ for all $n>0$.
} 
(ii) the KP-hierarchy

$$
\left(p_{k+4}(\tilde{\partial})-\frac{1}{2} \frac{\partial^{2}}{\partial t_{1} \partial t_{k+3}}\right) \tau_{n} \circ \tau_{n}=0,
$$

of which the first equation reads:

$$
\left(\left(\frac{\partial}{\partial t_{1}}\right)^{4}+3\left(\frac{\partial}{\partial t_{2}}\right)^{2}-4 \frac{\partial^{2}}{\partial t_{1} \partial t_{3}}\right) \log \tau_{n}+6\left(\frac{\partial^{2}}{\partial t_{1}^{2}} \log \tau_{n}\right)^{2}=0
$$

$$
k=0,1,2, \ldots
$$

(iii) The standard Toda lattice; i.e., the tridiagonal matrix

$$
L(t):=S(t) \Lambda S(t)^{-1}=\left(\begin{array}{cccc}
\frac{\partial}{\partial t_{1}} \log \frac{\tau_{1}}{\tau_{0}} & \left(\frac{\tau_{0} \tau_{2}}{\tau_{1}^{2}}\right)^{1 / 2} & 0 & \\
\left(\frac{\tau_{0} \tau_{2}}{\tau_{1}^{2}}\right)^{1 / 2} & \frac{\partial}{\partial t_{1}} \log \frac{\tau_{2}}{\tau_{1}} & \left(\frac{\tau_{1} \tau_{3}}{\tau_{2}^{2}}\right)^{1 / 2} & \\
0 & \left(\frac{\tau_{1} \tau_{3}}{\tau_{2}^{2}}\right)^{1 / 2} & \frac{\partial}{\partial t_{1}} \log \frac{\tau_{3}}{\tau_{2}} & \\
& & & \ddots
\end{array}\right)
$$

satisfies the commuting equations

$$
\frac{\partial L}{\partial t_{k}}=\left[\frac{1}{2}\left(L^{k}\right)_{\mathfrak{s}}, L\right] .
$$

(iv) Orthogonal polynomials: The $n^{\text {th }}$ degree polynomials $p_{n}(t ; z)$ in $z$, depending on $t \in \mathbb{C}^{\infty}$, orthonormal with respect to the $t$-dependent inner product (2.1.1)

$$
\left\langle p_{k}(t ; z), p_{\ell}(t ; z)\right\rangle=\delta_{k \ell}
$$

are eigenvectors of $L$, i.e., $(L(t) p(t ; z))_{n}=z p_{n}(t ; z), \quad n \geq 0$, and enjoy the following representations

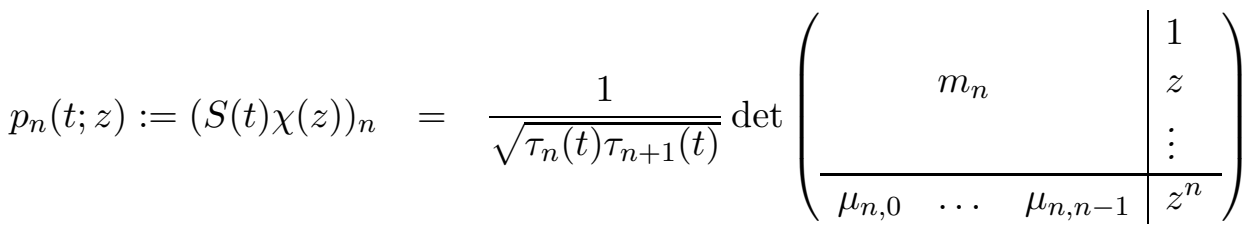

$$
\begin{aligned}
& =z^{n} h_{n}^{-1 / 2} \frac{\tau_{n}\left(t-\left[z^{-1}\right]\right)}{\tau_{n}(t)}, \quad h_{n}:=\frac{\tau_{n+1}(t)}{\tau_{n}(t)} .
\end{aligned}
$$

\footnotetext{
${ }^{6}$ for the customary Hirota symbol $p\left(\partial_{t}\right) f \circ g:=\left.p\left(\frac{\partial}{\partial y}\right) f(t+y) g(t-y)\right|_{y=0}$. The $p_{\ell}$ 's are the elementary Schur polynomials $e^{\sum_{1}^{\infty} t_{i} z^{i}}:=\sum_{i \geq 0} p_{i}\left(t_{1}, t_{2}, \ldots\right) z^{i}$ and $p_{\ell}(\tilde{\partial}):=p_{\ell}\left(\frac{\partial}{\partial t_{1}}, \frac{1}{2} \frac{\partial}{\partial t_{2}}, \ldots\right)$.

${ }^{7}()_{\mathfrak{s}}$ means: take the skew-symmetric part of () in the decomposition "skew-symmetric" + "lower-triangular."
} 
The functions $q_{n}(t ; z):=z \int_{\mathbb{R}^{n}} \frac{p_{n}(t ; u)}{z-u} \rho_{t}(u) d u$ are "dual eigenvectors" of $L$, i.e., $(L(t) q(t ; z))_{n}=z q_{n}(t ; z), \quad n \geq 1$, and have the following $\tau$-function representation: (see the remark at the end of this section)

$$
\begin{aligned}
q_{n}(t ; z):=z \int_{\mathbb{R}^{n}} \frac{p_{n}(t ; u)}{z-u} \rho_{t}(u) d u & =\left(S^{\top-1}(t) \chi\left(z^{-1}\right)\right)_{n} \\
& =\left(S(t) m_{\infty}(t) \chi\left(z^{-1}\right)\right)_{n} \\
& =z^{-n} h_{n}^{-1 / 2} \frac{\tau_{n+1}\left(t+\left[z^{-1}\right]\right)}{\tau_{n}(t)}
\end{aligned}
$$

(v) Bilinear relations: for all $n, m \geq 0$, and $a, b \in \mathbb{C}^{\infty}$, such that $a-b=$ $t-t^{\prime}$

$$
\begin{gathered}
\oint_{z=\infty} \tau_{n}\left(t-\left[z^{-1}\right]\right) \tau_{m+1}\left(t^{\prime}+\left[z^{-1}\right]\right) e^{\sum_{1}^{\infty} a_{i} z^{i}} z^{n-m-1} \frac{d z}{2 \pi i} \\
=\oint_{z=0} \tau_{n+1}(t+[z]) \tau_{m}\left(t^{\prime}-[z]\right) e^{\sum_{1}^{\infty} b_{i} z^{-i}} z^{n-m-1} \frac{d z}{2 \pi i} .
\end{gathered}
$$

In the case $\beta=2$, the Virasoro expressions take on a particularly elegant form, namely for $n \geq 0$,

$$
\begin{aligned}
\mathbb{J}_{k, n}^{(2)}(t) & =\sum_{i+j=k}: \mathbb{J}_{i, n}^{(1)}(t) \mathbb{J}_{j, n}^{(1)}(t):=J_{k}^{(2)}(t)+2 n J_{k}^{(1)}(t)+n^{2} \delta_{0 k} \\
\mathbb{J}_{k, n}^{(1)}(t) & =J_{k}^{(1)}(t)+n \delta_{0 k},
\end{aligned}
$$

with 8

$$
\begin{aligned}
J_{k}^{(1)} & =\frac{\partial}{\partial t_{k}}+\frac{1}{2}(-k) t_{-k}, \\
J_{k}^{(2)} & =\sum_{i+j=k} \frac{\partial^{2}}{\partial t_{i} \partial t_{j}}+\sum_{-i+j=k} i t_{i} \frac{\partial}{\partial t_{j}}+\frac{1}{4} \sum_{-i-j=k} i t_{i} j t_{j} .
\end{aligned}
$$

Statement (i) is already contained in Theorem 1.1, whereas the other statements can be found in [1], [2], and [5]. Notice that the standard Toda lattice is a reduction of the semi-infinite 2-Toda lattice, where $\tau_{n}(t, s)=\tau_{n}(t-s)$. The 2-Toda lattice arises in the context of a factorization of a generic semiinfinite matrix $m_{\infty}(t, s)$, satisfying the simple equations $\frac{\partial m_{\infty}}{\partial t_{k}}=\Lambda^{k} m_{\infty}, \frac{\partial m_{\infty}}{\partial s_{k}}=$ $-m_{\infty} \Lambda^{\top k}$, whereas the standard Toda lattice is related to the same factorization of $m_{\infty}(t, s)$, but where $m_{\infty}(t, s)$ is Hänkel (i.e., $\Lambda m_{\infty}=m_{\infty} \Lambda^{\top}$ ).

\footnotetext{
${ }^{8}$ The expression $J_{k}^{(1)}=0$ for $k=0$.
} 
Remark. The vectors $p$ and $q$ are eigenvectors of $L$. Indeed, remembering $\chi(z)=\left(1, z, z^{2}, \ldots\right)^{\top}$, we have

$$
\Lambda \chi(z)=z \chi(z) \text { and } \Lambda^{\top} \chi\left(z^{-1}\right)=z \chi\left(z^{-1}\right)-z e_{1}, \quad \text { with } e_{1}=(1,0,0, \ldots)^{\top} .
$$

Therefore, $p(z)=S \chi(z)$ and $q(z)=S^{\top-1} \chi\left(z^{-1}\right)$ are eigenvectors, in the sense

$$
\begin{aligned}
L p & =S \Lambda S^{-1} S \chi(z)=z S \chi(z)=z p, \\
L^{\top} q & =S^{\top-1} \Lambda^{\top} S^{\top} S^{\top-1} \chi\left(z^{-1}\right) \\
& =z S^{\top-1} \chi\left(z^{-1}\right)-z S^{\top-1} e_{1}=z q-z S^{\top-1} e_{1} .
\end{aligned}
$$

Then, using $L=L^{\top}$, one is lead to

$$
((L-z I) p)_{n}=0, \text { for } n \geq 0 \quad \text { and } \quad((L-z I) q)_{n}=0, \text { for } n \geq 1 .
$$

2.2. Symmetric/symplectic matrix integrals and the Pfaff lattice. Consider an inner-product, with a skew-symmetric weight $\rho(y, z)$,

$$
\langle f, g\rangle_{t}=\iint_{\mathbb{R}^{2}} f(y) g(z) e^{\sum_{1}^{\infty} t_{i}\left(y^{i}+z^{i}\right)} \rho(y, z) d y d z, \text { with } \rho(z, y)=-\rho(y, z) .
$$

Then, since

$$
\langle f, g\rangle_{t}=-\langle g, f\rangle_{t}
$$

the (semi-infinite) moment matrix, depending on $t=\left(t_{1}, t_{2}, \ldots\right)$,

$$
m_{n}(t)=\left(\mu_{i j}(t)\right)_{0 \leq i, j \leq n-1}=\left(\left\langle y^{i}, z^{j}\right\rangle_{t}\right)_{0 \leq i, j \leq n-1}
$$

is skew-symmetric and the semi-infinite matrix $m_{\infty}$ evolves in $t$ according to the commuting vector fields

$$
\frac{\partial \mu_{i j}}{\partial t_{k}}=\mu_{i+k, j}+\mu_{i, j+k} \text {, i.e., } \frac{\partial m_{\infty}}{\partial t_{k}}=\Lambda^{k} m_{\infty}+m_{\infty} \Lambda^{\top k} .
$$

It is well known that the determinant of an odd skew-symmetric matrix equals 0 , whereas the determinant of an even skew-symmetric matrix is the square of a polynomial in the entries, the Pfaffian, with a sign specified below. So

$$
\begin{aligned}
\operatorname{det}\left(m_{2 n-1}(t)\right) & =0 \\
\left(\operatorname{det} m_{2 n}(t)\right)^{1 / 2}= & p f\left(m_{2 n}(t)\right)=\frac{1}{n !}\left(d x_{0} \wedge d x_{1} \wedge \ldots \wedge d x_{2 n-1}\right)^{-1} \\
& \left(\sum_{0 \leq i<j \leq 2 n-1} \mu_{i j}(t) d x_{i} \wedge d x_{j}\right)^{n} .
\end{aligned}
$$

Define now the Pfaffian $\tau$-functions:

$$
\tau_{2 n}(t):=p f m_{2 n}(t),
$$


and the semi-infinite skew-symmetric matrix, 0 everywhere, except for the $2 \times 2$ blocks, along the diagonal:

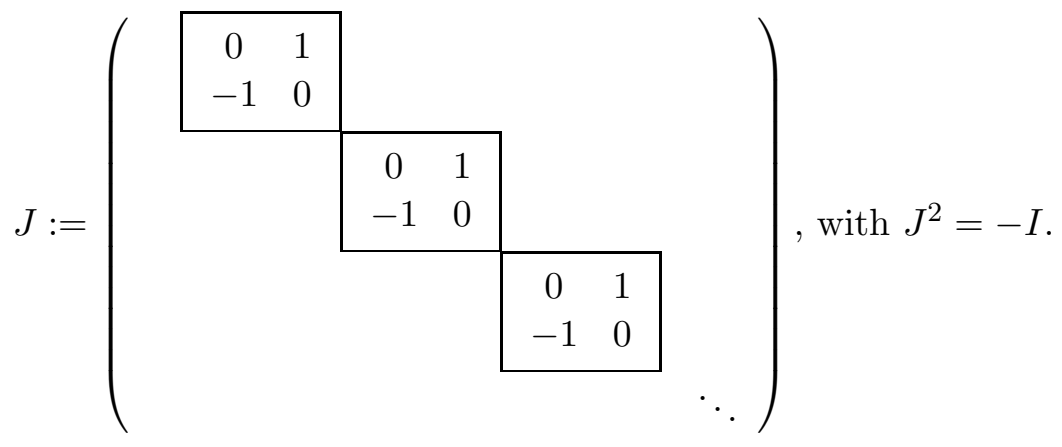

Since $m_{\infty}$ is skew-symmetric, $m_{\infty}$ does not admit a Borel factorization in the standard sense, but $m_{\infty}$ admits a unique factorization, with the matrix $J$ inserted (see [6]):

$$
m_{\infty}(t)=Q^{-1}(t) J Q^{\top-1}(t)
$$

where

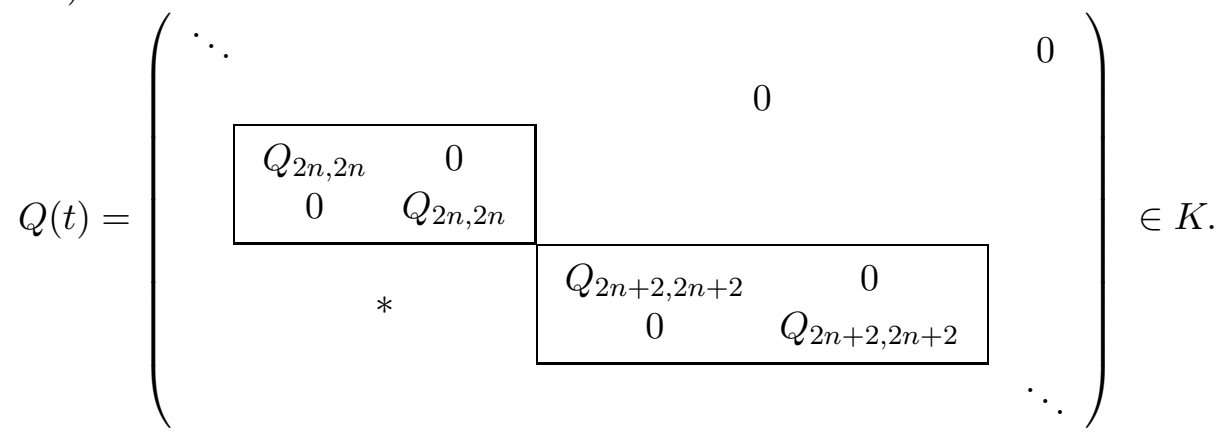

$K$ is the group of lower-triangular invertible matrices of the form above, with Lie algebra $\mathfrak{k}$ of matrices of precisely the same form. In this problem, the Lie algebra splitting of semi-infinite matrices is given by

$$
g l(\infty)=\mathfrak{k} \oplus \mathfrak{n}\left\{\begin{array}{l}
\mathfrak{k}=\{\text { lower-triangular matrices of the form }(2.2 .5)\} \\
\mathfrak{n}=s p(\infty)=\left\{a \text { such that } J a^{\top} J=a\right\}
\end{array}\right.
$$

with unique decomposition ( $a_{ \pm}$refers to projection onto strictly upper- (strictly lower) triangular matrices, with all $2 \times 2$ diagonal blocks equal to zero)

$$
\begin{aligned}
a=(a)_{\mathfrak{k}}+ & (a)_{\mathfrak{n}} \\
=\quad\left(\left(a_{-}-\right.\right. & \left.\left.J\left(a_{+}\right)^{\top} J\right)+\frac{1}{2}\left(a_{0}-J\left(a_{0}\right)^{\top} J\right)\right) \\
& \quad+\left(\left(a_{+}+J\left(a_{+}\right)^{\top} J\right)+\frac{1}{2}\left(a_{0}+J\left(a_{0}\right)^{\top} J\right)\right) .
\end{aligned}
$$


Considering as a special skew-symmetric weight (2.2.1),

$$
\rho(y, z):=2 D^{\alpha} \delta(y-z) \tilde{\rho}(y) \tilde{\rho}(z), \text { with } \alpha=\mp 1, \quad \tilde{\rho}(y)=e^{-\tilde{V}(y)},
$$

the inner-product (2.2.1) becomes

$$
\begin{aligned}
\langle f, g\rangle_{t} & =\iint_{\mathbb{R}^{2}} f(y) g(z) e^{\sum t_{i}\left(y^{i}+z^{i}\right)} 2 D^{\alpha} \delta(y-z) \tilde{\rho}(y) \tilde{\rho}(z) d y d z \\
& = \begin{cases}\iint_{\mathbb{R}^{2}} f(y) g(z) e^{\sum_{1}^{\infty} t_{i}\left(y^{i}+z^{i}\right)} \varepsilon(y-z) \tilde{\rho}(y) \tilde{\rho}(z) d y d z, & \text { for } \alpha=-1 \\
\int_{\mathbb{R}}\{f, g\}(y) e^{\sum_{1}^{\infty} 2 t_{i} y^{i}} \tilde{\rho}(y)^{2} d y, & \text { for } \alpha=+1,\end{cases}
\end{aligned}
$$

and (see $[16],[4])$

$$
\begin{aligned}
& p f\left(\left\langle y^{i}, z^{j}\right\rangle_{t}\right)_{0 \leq i, j \leq 2 n-1}
\end{aligned}
$$

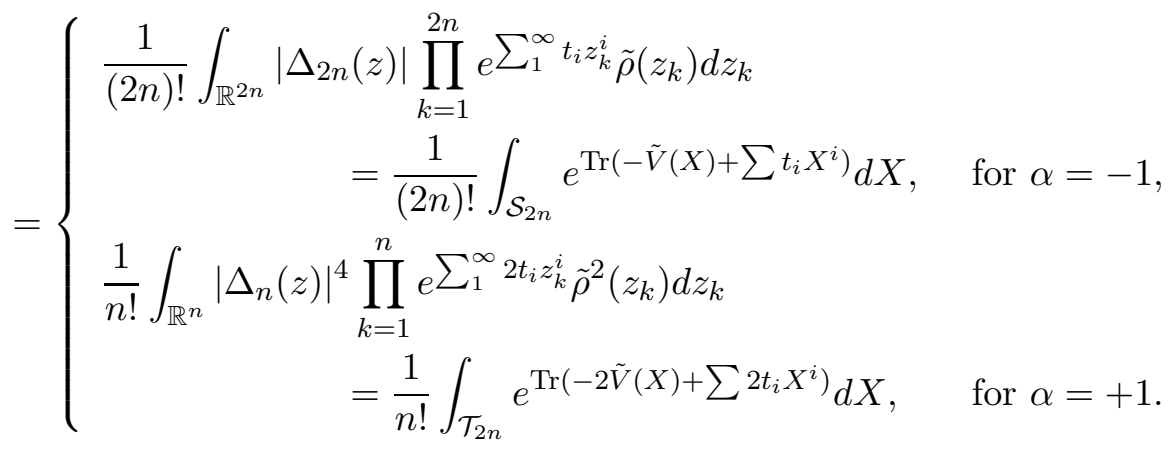

Setting

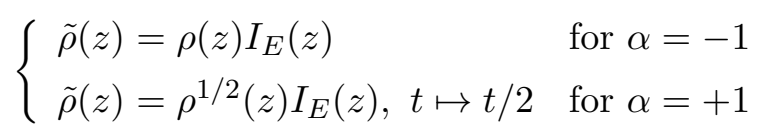

in the identities (2.2.9), we are led to the identities between integrals and Pfaffians, which are spelled out in Theorem 2.2:

Theorem 2.2. The integrals $I_{n}(t, c)$,

$$
I_{n}=\int_{E^{n}}\left|\Delta_{n}(z)\right|^{\beta} \prod_{k=1}^{n}\left(e^{\sum_{1}^{\infty} t_{i} z_{k}^{i}} \rho\left(z_{k}\right) d z_{k}\right)
$$

\footnotetext{
${ }^{9} \varepsilon(y)=\operatorname{sign}(y)$, and $\{f, g\}:=f^{\prime} g-f g^{\prime}$. Also notice that $\varepsilon^{\prime}=2 \delta(x)$.
} 


$$
=\left\{\begin{array}{cc}
n ! p f\left(\iint_{E^{2}} y^{i} z^{j} \varepsilon(y-z) e^{\sum_{1}^{\infty} t_{k}\left(y^{k}+z^{k}\right)} \rho(y) \rho(z) d y d z\right)_{0 \leq i, j \leq n-1} \\
=n ! \tau_{n}(t, c), & n \text { even, for } \beta=1 \\
n ! p f\left(\int_{E}\left\{y^{i}, y^{j}\right\} e^{\sum_{1}^{\infty} t_{k} y^{k}} \rho(y) d y\right)_{0 \leq i, j \leq 2 n-1} & =n ! \tau_{2 n}(t / 2, c), \\
& n \text { arbitrary, for } \beta=4
\end{array}\right.
$$

and the $\tau_{n}(t, c)$ 's above satisfy the following equations:

(i) The Virasoro constraints 10 (1.1.4) for $\beta=1,4$,

$$
\left(-\sum_{1}^{2 r} c_{i}^{k+1} f\left(c_{i}\right) \frac{\partial}{\partial c_{i}}+\sum_{i \geq 0}\left(a_{i}{ }^{\beta} \mathbb{J}_{k+i, n}^{(2)}-b_{i}{ }^{\beta} \mathbb{J}_{k+i+1, n}^{(1)}\right)\right) I_{n}=0
$$

(ii) The Pfaff-KP hierarchy: (see footnote 6 )

$$
\begin{array}{r}
\left(p_{k+4}(\tilde{\partial})-\frac{1}{2} \frac{\partial^{2}}{\partial t_{1} \partial t_{k+3}}\right) \tau_{n} \circ \tau_{n}=p_{k}(\tilde{\partial}) \tau_{n+2} \circ \tau_{n-2} \\
n \text { even, } k=0,1,2, \ldots .
\end{array}
$$

of which the first equation reads

$$
\left(\left(\frac{\partial}{\partial t_{1}}\right)^{4}+3\left(\frac{\partial}{\partial t_{2}}\right)^{2}-4 \frac{\partial^{2}}{\partial t_{1} \partial t_{3}}\right) \log \tau_{n}+6\left(\frac{\partial^{2}}{\partial t_{1}^{2}} \log \tau_{n}\right)^{2}=12 \frac{\tau_{n-2} \tau_{n+2}}{\tau_{n}^{2}}
$$

$n$ even.

(iii) The Pfaff lattice: The time-dependent matrix

$$
L(t)=Q(t) \Lambda Q(t)^{-1}
$$

satisfies the Hamiltonian commuting equations, given by the AdlerKostant-Symes splitting theorem, applied to the splitting $g l(\infty)=\mathfrak{k} \oplus \mathfrak{n}$, as in (2.2.6) and (2.2.7),

$$
\frac{\partial L}{\partial t_{i}}=\left[-\left(L^{i}\right)_{\mathfrak{k}}, L\right], \quad \text { (Pfaff lattice) }
$$

(iv) Skew-orthogonal polynomials: The vector of time-dependent polynomials $q(t ; z):=\left(q_{n}(t ; z)\right)_{n \geq 0}=Q(t) \chi(z)$ in $z$ satisfy the eigenvalue problem

$$
L(t) q(t, z)=z q(t, z)
$$

\footnotetext{
${ }^{10}$ here the $a_{i}$ 's and $b_{i}$ 's are defined in the usual way, in terms of $\rho(z)$; namely, $-\frac{\rho^{\prime}}{\rho}=\frac{\sum b_{i} z^{i}}{\sum a_{i} z^{i}}$.
} 
and enjoy the following representations:

$$
\begin{aligned}
q_{2 n}(t ; z) & =z^{2 n} h_{2 n}^{-1 / 2} \frac{\tau_{2 n}\left(t-\left[z^{-1}\right]\right)}{\tau_{2 n}(t)}, \quad h_{2 n}=\frac{\tau_{2 n+2}(t)}{\tau_{2 n}(t)} \\
q_{2 n+1}(t ; z) & =z^{2 n} h_{2 n}^{-1 / 2} \frac{1}{\tau_{2 n}(t)}\left(z+\frac{\partial}{\partial t_{1}}\right) \tau_{2 n}\left(t-\left[z^{-1}\right]\right) .
\end{aligned}
$$

They are skew-orthogonal polynomials in z; i.e.,

$$
\left\langle q_{i}(t ; z), q_{j}(t ; z)\right\rangle_{t}=J_{i j} .
$$

(v) The bilinear identities: For all $n, m \geq 0$, the $\tau_{2 n}$ 's satisfy the following bilinear identity

$$
\begin{aligned}
& \oint_{z=\infty} \tau_{2 n}\left(t-\left[z^{-1}\right]\right) \tau_{2 m+2}\left(t^{\prime}+\left[z^{-1}\right]\right) e^{\sum_{1}^{\infty}\left(t_{i}-t_{i}^{\prime}\right) z^{i}} z^{2 n-2 m-2} \frac{d z}{2 \pi i} \\
& \quad+\oint_{z=0} \tau_{2 n+2}(t+[z]) \tau_{2 m}\left(t^{\prime}-[z]\right) e^{\sum_{1}^{\infty}\left(t_{i}^{\prime}-t_{i}\right) z^{-i}} z^{2 n-2 m} \frac{d z}{2 \pi i}=0
\end{aligned}
$$

Note that (2.2.10) is a consequence of Theorem 1.1, while items (ii) to (v) are shown in [4], [6]. (See [8] for the Pfaff lattice, viewed as a reduction of the 2-Toda lattice.) A semi-infinite matrix $m_{\infty}(t, s)$, satisfying $\frac{\partial m_{\infty}}{\partial s_{k}}=$ $\Lambda^{k} m_{\infty}, \frac{\partial m_{\infty}}{\partial t_{k}}=-m_{\infty} \Lambda^{\top k}$, leads to the semi-infinite 2-Toda lattice. When the initial condition $m_{\infty}(0,0)$ is skew-symmetric, then $m_{\infty}(t,-t)$ remains skewsymmetric in time and $\tau_{n}(t)=\left(\tau_{n}(t,-t)\right)^{1 / 2}=p f m_{n}(t,-t)$ is a Pfaff lattice $\tau$-function.

\section{Expressing $t$-partials in terms of boundary-partials}

3.1. Gaussian and Laguerre ensembles. Given first-order linear operators $\mathcal{D}_{1}, \mathcal{D}_{2}, \mathcal{D}_{3}$ in $c=\left(c_{1}, \ldots, c_{2 r}\right) \in \mathbb{R}^{2 r}$ and a function $F(t, c)$, with $t \in \mathbb{C}^{\infty}$, satisfying the following partial differential equations in $t$ and $c$ :

$$
\mathcal{D}_{k} F=\frac{\partial F}{\partial t_{k}}+\sum_{-1 \leq j<k} \gamma_{k j} V_{j}(F)+\gamma_{k}+\delta_{k} t_{1}, \quad k=1,2,3, \ldots,
$$

with $V_{j}(F)$ nonlinear differential operators in $t_{i}$ of which the first few are given here:

$$
V_{j}(F)=\sum_{i, i+j \geq 1} i t_{i} \frac{\partial F}{\partial t_{i+j}}+\frac{\beta}{2} \delta_{2, j}\left(\frac{\partial^{2} F}{\partial t_{1}^{2}}+\left(\frac{\partial F}{\partial t_{1}}\right)^{2}\right), \quad-1 \leq j \leq 2 .
$$

In (3.1.1) and (3.1.2), $\beta>0, \gamma_{k j}, \gamma_{k}, \delta_{k}$ are arbitrary parameters; also $\delta_{2 j}=0$ for $j \neq 2$ and $\delta_{2 j}=1$ for $j=2$. The claim is that the equations (3.1.1) enable 
one to express all partial derivatives,

$$
\left.\frac{\partial^{i_{1}+\ldots+i_{k}} F(t, c)}{\partial t_{1}^{i_{1}} \ldots \partial t_{k}^{i_{k}}}\right|_{\mathcal{L}}, \text { along } \mathcal{L}:=\left\{\text { all } t_{i}=0, c=\left(c_{1}, \ldots, c_{2 r}\right) \text { arbitrary }\right\},
$$

uniquely in terms of polynomials in $\mathcal{D}_{j_{1}} \ldots \mathcal{D}_{j_{r}} F(0, c)$. Indeed, the method consists of expressing $\left.\frac{\partial F}{\partial t_{k}}\right|_{t=0}$ in terms of $\left.\mathcal{D}_{k} f\right|_{t=0}$, using (3.1.1). Second derivatives are obtained by acting on $\mathcal{D}_{k} F$ with $\mathcal{D}_{\ell}$, by noting that $\mathcal{D}_{\ell}$ commutes with all $t$-derivatives, by using the equation for $\mathcal{D}_{\ell} F$, and by setting in the end $t=0$ :

$$
\begin{aligned}
\mathcal{D}_{\ell} \mathcal{D}_{k} F & =\mathcal{D}_{\ell} \frac{\partial F}{\partial t_{k}}+\sum_{-1 \leq j<k} \gamma_{k j} \mathcal{D}_{\ell}\left(V_{j}(F)\right) \\
& =\left(\frac{\partial}{\partial t_{k}}+\sum_{-1 \leq j<k} \gamma_{k j} V_{j}\right) \mathcal{D}_{\ell}(F), \quad \text { provided } V_{j}(F) \text { does not } \\
& =\left(\frac{\partial}{\partial t_{k}}+\sum_{-1 \leq j<k} \gamma_{k j} V_{j}\right)\left(\frac{\partial F}{\partial t_{\ell}}+\sum_{-1 \leq j<\ell} \gamma_{\ell j} V_{j}(F)+\delta_{\ell} t_{1}\right) \\
& =\frac{\partial^{2} F}{\partial t_{k} \partial t_{\ell}}+\text { lower-weight terms. }
\end{aligned}
$$

When the nonlinear term is present, it is taken care of as follows:

$$
\begin{aligned}
\mathcal{D}_{\ell}\left(\frac{\partial F}{\partial t_{1}}\right)^{2} & =2 \frac{\partial F}{\partial t_{1}} \mathcal{D}_{\ell} \frac{\partial F}{\partial t_{1}} \\
& =2 \frac{\partial F}{\partial t_{1}} \frac{\partial}{\partial t_{1}} \mathcal{D}_{\ell} F \\
& =2 \frac{\partial F}{\partial t_{1}} \frac{\partial}{\partial t_{1}}\left(\frac{\partial F}{\partial t_{\ell}}+\sum_{-1 \leq j<\ell} \gamma_{\ell j} V_{j}(F)+\gamma_{\ell}+\delta_{\ell} t_{1}\right)
\end{aligned}
$$

higher derivatives are obtained in the same way. Explicit expressions for only a few partials, useful in the next subsection, will be given here:

$$
\begin{aligned}
\left.\frac{\partial F}{\partial t_{1}}\right|_{\mathcal{L}} & =\mathcal{D}_{1} F-\gamma_{1} \\
\left.\frac{\partial^{2} F}{\partial t_{1}^{2}}\right|_{\mathcal{L}} & =\left(\mathcal{D}_{1}^{2}-\gamma_{10} \mathcal{D}_{1}\right) F+\gamma_{10} \gamma_{1}-\delta_{1}
\end{aligned}
$$




$$
\begin{aligned}
& \left.\frac{\partial^{3} F}{\partial t_{1}^{3}}\right|_{\mathcal{L}}=\left(\mathcal{D}_{1}^{3}-3 \gamma_{10} \mathcal{D}_{1}^{2}+2 \gamma_{10}^{2} \mathcal{D}_{1}\right) F+2 \gamma_{10}\left(\delta_{1}-\gamma_{1} \gamma_{10}\right) \\
& \left.\frac{\partial^{4} F}{\partial t_{1}^{4}}\right|_{\mathcal{L}}=\left(\mathcal{D}_{1}^{4}-6 \gamma_{10} \mathcal{D}_{1}^{3}+11 \gamma_{10}^{2} \mathcal{D}_{1}^{2}-6 \gamma_{10}^{3} \mathcal{D}_{1}\right) F-6 \gamma_{10}^{2}\left(\delta_{1}-\gamma_{1} \gamma_{10}\right), \\
& \left.\frac{\partial F}{\partial t_{2}}\right|_{\mathcal{L}}=\mathcal{D}_{2} F-\gamma_{2}, \\
& \left.\frac{\partial^{2} F}{\partial t_{2}^{2}}\right|_{\mathcal{L}}=\left(\mathcal{D}_{2}^{2}-2 \gamma_{20} \mathcal{D}_{2}+\beta \gamma_{21} \gamma_{32} \mathcal{D}_{1}^{2}\right. \\
& \left.-\left(\left(2 \gamma_{1}+\gamma_{10}\right) \gamma_{21} \gamma_{32} \beta+2 \gamma_{2,-1}\right) \mathcal{D}_{1}-2 \gamma_{21} \mathcal{D}_{3}\right) F \\
& +\beta \gamma_{21} \gamma_{32}\left(\mathcal{D}_{1} F\right)^{2}+\beta \gamma_{21} \gamma_{32}\left(\gamma_{1}^{2}+\gamma_{10} \gamma_{1}-\delta_{1}\right) \\
& +2\left(\gamma_{21} \gamma_{3}+\gamma_{20} \gamma_{2}+\gamma_{1} \gamma_{2,-1}\right) \text {, } \\
& \left.\frac{\partial F}{\partial t_{3}}\right|_{\mathcal{L}}=\left(\mathcal{D}_{3}-\frac{\beta}{2} \gamma_{32} \mathcal{D}_{1}^{2}+\frac{\beta}{2} \gamma_{32}\left(2 \gamma_{1}+\gamma_{10}\right) \mathcal{D}_{1}\right) F-\frac{\beta}{2} \gamma_{32}\left(\mathcal{D}_{1} F\right)^{2} \\
& +\frac{\beta}{2} \gamma_{32}\left(\delta_{1}-\gamma_{1} \gamma_{10}-\gamma_{1}^{2}\right)-\gamma_{3}, \\
& \left.\frac{\partial^{2} F}{\partial t_{1} \partial t_{3}}\right|_{\mathcal{L}}=\left(\mathcal{D}_{1} \mathcal{D}_{3}-\frac{\beta}{2} \gamma_{32} \mathcal{D}_{1}^{3}+\beta \gamma_{32}\left(\gamma_{1}+2 \gamma_{10}\right) \mathcal{D}_{1}^{2}\right. \\
& \left.-\frac{3 \beta}{2} \gamma_{10} \gamma_{32}\left(2 \gamma_{1}+\gamma_{10}\right) \mathcal{D}_{1}-3 \gamma_{1,-1} \mathcal{D}_{2}-3 \gamma_{10} \mathcal{D}_{3}\right) F \\
& +\frac{3 \beta}{2} \gamma_{10} \gamma_{32}\left(\mathcal{D}_{1} F\right)^{2}-\beta \gamma_{32}\left(\mathcal{D}_{1} F\right)\left(\mathcal{D}_{1}^{2} F\right) \\
& +\frac{3}{2}\left(2 \gamma_{10} \gamma_{3}+\beta \gamma_{32} \gamma_{10}\left(\gamma_{1}^{2}+\gamma_{10} \gamma_{1}-\delta_{1}\right)+2 \gamma_{1,-1} \gamma_{2}\right)
\end{aligned}
$$

3.2. Jacobi ensemble.

1. From the expressions (1.3.11), upon evaluating $\left.\mathcal{B}_{-1} F\right|_{t=0},\left.\mathcal{B}_{-1}^{2} F\right|_{t=0}$, $\left.\mathcal{B}_{0} F\right|_{t=0}$, one finds the following equations, both sides of which are evaluated at $t=0$,

$$
\begin{aligned}
-\mathcal{B}_{-1} F & =\sigma_{1} \frac{\partial}{\partial t_{1}} F+b_{0} n \\
\frac{1}{\sigma_{1}} \mathcal{B}_{-1}^{2} F & =\left(\sigma_{1} \frac{\partial^{2}}{\partial t_{1}{ }^{2}}+\frac{\partial}{\partial t_{2}}\right) F-n
\end{aligned}
$$




$$
-\mathcal{B}_{0} F=\left(b_{0} \frac{\partial}{\partial t_{1}}+\sigma_{2} \frac{\partial}{\partial t_{2}}\right) F+\frac{\beta}{2}\left(\left(\frac{\partial}{\partial t_{1}}\right)^{2} F+\left(\frac{\partial F}{\partial t_{1}}\right)^{2}\right)-\frac{n}{2}\left(\sigma_{1}-b_{1}\right) .
$$

From these expressions, one extracts

$$
\left.\frac{\partial F}{\partial t_{1}}\right|_{t=0},\left.\frac{\partial^{2} F}{\partial t_{1}^{2}}\right|_{t=0},\left.\frac{\partial F}{\partial t_{2}}\right|_{t=0}
$$

in terms of $\mathcal{B}_{j}^{i} F$.

2. From the expressions for $\left.\mathcal{B}_{-1}^{3} F\right|_{t=0},\left.\mathcal{B}_{0} \mathcal{B}_{-1} F\right|_{t=0},\left.\mathcal{B}_{1} F\right|_{t=0}$, namely

$$
\begin{aligned}
\mathcal{B}_{1} F & =\left(-b_{0} \frac{\partial}{\partial t_{2}}+\left(\sigma_{1}-b_{1}\right) \frac{\partial}{\partial t_{1}}-\sigma_{3} \frac{\partial}{\partial t_{3}}\right) F-\beta\left(\frac{\partial^{2} F}{\partial t_{1} \partial t_{2}}+\frac{\partial F}{\partial t_{1}} \frac{\partial F}{\partial t_{2}}\right) \\
\frac{1}{\sigma_{1}} \mathcal{B}_{0} \mathcal{B}_{-1} F & =\left(\sigma_{2} \frac{\partial^{2}}{\partial t_{1} \partial t_{2}}+\frac{\partial}{\partial t_{3}}-\frac{\partial}{\partial t_{1}}+b_{0} \frac{\partial^{2}}{\partial t_{1}^{2}}\right) F+\frac{\beta}{2}\left(\frac{\partial^{3} F}{\partial t_{1}^{3}}+2 \frac{\partial F}{\partial t_{1}} \frac{\partial^{2} F}{\partial t_{1}^{2}}\right) \\
-\frac{1}{\sigma_{1}} \mathcal{B}_{-1}^{3} F & =\left(\sigma_{1}^{2} \frac{\partial^{3}}{\partial t_{1}^{3}}+3 \sigma_{1} \frac{\partial^{2}}{\partial t_{1} \partial t_{2}}-2 \frac{\partial}{\partial t_{1}}+2 \frac{\partial}{\partial t_{3}}\right) F
\end{aligned}
$$

one extracts

$$
\left.\frac{\partial F}{\partial t_{3}}\right|_{t=0},\left.\frac{\partial^{2} F}{\partial t_{1}^{3}}\right|_{t=0},\left.\frac{\partial^{2} F}{\partial t_{1} \partial t_{2}}\right|_{t=0}
$$

in terms of $\mathcal{B}_{j}^{i} F$, using the previous extractions.

3. From the expressions for $\left.\mathcal{B}_{2} F\right|_{t=0},\left.\mathcal{B}_{1} \mathcal{B}_{-1} F\right|_{t=0},\left.\mathcal{B}_{0}^{2} F\right|_{t=0},\left.\mathcal{B}_{0} \mathcal{B}_{-1}^{2} F\right|_{t=0}$, $\left.\mathcal{B}_{-1}^{4} F\right|_{t=0}$, namely, (where both sides are evaluated at $t=0$ )

$$
\begin{aligned}
\mathcal{B}_{2} F= & \left(-\sigma_{4} \frac{\partial}{\partial t_{4}}-b_{0} \frac{\partial}{\partial t_{3}}+\left(\sigma_{2}-b_{1}\right) \frac{\partial}{\partial t_{2}}+\frac{\beta}{2}\left(\frac{\partial^{2}}{\partial t_{1}^{2}}-\frac{\partial^{2}}{\partial t_{2}^{2}}-2 \frac{\partial^{2}}{\partial t_{1} \partial t_{3}}\right)\right) F \\
& +\beta\left(\left(\frac{\partial F}{\partial t_{1}}\right)^{2}-\left(\frac{\partial F}{\partial t_{2}}\right)^{2}-\frac{\partial F}{\partial t_{1}} \frac{\partial F}{\partial t_{3}}\right) \\
\frac{1}{\sigma_{1}} \mathcal{B}_{1} \mathcal{B}_{-1} F= & \left(\frac{\partial}{\partial t_{4}}-\frac{\partial}{\partial t_{2}}+b_{0} \frac{\partial^{2}}{\partial t_{1} \partial t_{2}}+\sigma_{3} \frac{\partial^{2}}{\partial t_{1} \partial t_{3}}-\left(\sigma_{1}-b_{1}\right) \frac{\partial^{2}}{\partial t_{1}^{2}}+\beta \frac{\partial^{3}}{\partial t_{1}^{2} \partial t_{2}}\right) F \\
& +\beta\left(\frac{\partial^{2} F}{\partial t_{1}^{2}} \frac{\partial F}{\partial t_{2}}+\frac{\partial F}{\partial t_{1}} \frac{\partial^{2} F}{\partial t_{1} \partial t_{2}}\right) \\
\mathcal{B}_{0}^{2} F= & \left(b_{0} \frac{\partial}{\partial t_{1}}+\sigma_{2} \frac{\partial}{\partial t_{2}}+\frac{\beta}{2} \frac{\partial^{2}}{\partial t_{1}^{2}}+\beta \frac{\partial}{\partial t_{1}} F \frac{\partial}{\partial t_{1}}\right) \\
& \left(b_{0} \frac{\partial F}{\partial t_{1}}+\sigma_{2} \frac{\partial F}{\partial t_{2}}+\sum_{1}^{2} i t_{i}\left(\frac{\partial F}{\partial t_{i+2}}-\frac{\partial F}{\partial t_{i}}\right)+\frac{\beta}{2}\left(\frac{\partial^{2} F}{\partial t_{1}^{2}}+\left(\frac{\partial F}{\partial t_{1}}\right)^{2}\right)\right)
\end{aligned}
$$




$$
\begin{aligned}
\frac{1}{\sigma_{1}} \mathcal{B}_{0} \mathcal{B}_{-1}^{2} F= & -\frac{\partial}{\partial t_{1}}\left(\sigma_{1} \frac{\partial}{\partial t_{1}}+t_{1} \frac{\partial}{\partial t_{2}}\right) \\
& \left(b_{0} \frac{\partial F}{\partial t_{1}}+\sigma_{2} \frac{\partial F}{\partial t_{2}}+\sum_{1}^{2} i t_{i}\left(\frac{\partial F}{\partial t_{i+2}}-\frac{\partial F}{\partial t_{i}}\right)+\frac{\beta}{2}\left(\frac{\partial^{2} F}{\partial t_{1}^{2}}+\left(\frac{\partial F}{\partial t_{1}}\right)^{2}\right)\right), \\
\mathcal{B}_{-1}^{4} F= & \sigma_{1} \frac{\partial}{\partial t_{1}}\left(\sigma_{1} \frac{\partial}{\partial t_{1}}+t_{1} \frac{\partial}{\partial t_{2}}\right)\left(\sigma_{1} \frac{\partial}{\partial t_{1}}+t_{1} \frac{\partial}{\partial t_{2}}+2 t_{2}\left(\frac{\partial}{\partial t_{3}}-\frac{\partial}{\partial t_{1}}\right)\right) \\
& \left(\sigma_{1} \frac{\partial F}{\partial t_{1}}+t_{1} \frac{\partial F}{\partial t_{2}}+\sum_{2}^{3} i t_{i}\left(\frac{\partial F}{\partial t_{i+1}}-\frac{\partial F}{\partial t_{i-1}}\right)+b_{0} n-n t_{1}\right)
\end{aligned}
$$

one extracts

$$
\left.\frac{\partial^{4} F}{\partial t_{1}^{4}}\right|_{t=0},\left.\frac{\partial F}{\partial t_{4}}\right|_{t=0},\left.\frac{\partial^{3} F}{\partial t_{1}^{2} \partial t_{2}}\right|_{t=0},\left.\frac{\partial^{2} F}{\partial t_{1} \partial t_{3}}\right|_{t=0},\left.\frac{\partial^{2} F}{\partial t_{2}^{2}}\right|_{t=0},
$$

again in terms of $\mathcal{B}_{j}^{i} F$, using all the previous extractions.

3.3. Evaluating the matrix integrals on the full range. The denominators of the probabilities (0.0.4), for $\beta=1$, 4 ; namely:

$$
I_{n}^{(\beta)}:=\left\{\begin{array}{l}
\int_{\mathbb{R}^{n}}\left|\Delta_{n}(z)\right|^{\beta} \prod_{k=1}^{n} e^{-b z_{k}^{2}} d z_{k} \\
\int_{\mathbb{R}_{+}^{n}}\left|\Delta_{n}(z)\right|^{\beta} \prod_{k=1}^{n} z_{k}^{a} e^{-b z_{k}} d z_{k} \\
\int_{[-1,1]^{n}}\left|\Delta_{n}(z)\right|^{\beta} \prod_{k=1}^{n}\left(1-z_{k}\right)^{a}\left(1+z_{k}\right)^{b} d z_{k}
\end{array},\right.
$$

can be evaluated, using Selberg's integral (see Mehta [16, p. 340]):

$$
I_{n}^{(\beta)}\left\{\begin{array}{c}
=(2 \pi)^{n / 2}(2 b)^{-n(\beta(n-1)+2) / 4} \prod_{j=0}^{n-1} \frac{\Gamma((j+1) \beta / 2+1)}{\Gamma(\beta / 2+1)} \\
=b^{-n(\beta(n-1)+2 a+2) / 2} \prod_{j=0}^{n-1} \frac{\Gamma(a+1+j \beta / 2) \Gamma((j+1) \beta / 2+1)}{\Gamma(\beta / 2+1)} \\
=2^{n(2 a+2 b+\beta(n-1)+2) / 2} \\
\cdot \prod_{j=0}^{n-1} \frac{\Gamma(a+j \beta / 2+1) \Gamma(b+j \beta / 2+1) \Gamma((j+1) \beta / 2+1)}{\Gamma(\beta / 2+1) \Gamma(a+b+(n+j-1) \beta / 2+2)}
\end{array}\right.
$$

Lemma 3.1. For future use, the following expressions

$$
b_{n}^{(\beta=1)}:=\frac{(n !)^{2}}{(n-2) !(n+2) !} \frac{I_{n-2}^{(1)} I_{n+2}^{(1)}}{\left(I_{n}^{(1)}\right)^{2}}= \begin{cases}\frac{n(n-1)}{16 b^{2}} \quad \text { (Gauss) } \\ \frac{n(n-1)(n+2 a)(n+2 a+1)}{16 b^{4}} \text { (Laguerre) } \\ \frac{Q}{Q_{6}^{ \pm}} & \text {(Jacobi) }\end{cases}
$$




$$
b_{n}^{(\beta=4)}:=\frac{(n !)^{2}}{(n-1) !(n+1) !} \frac{I_{n-1}^{(4)} I_{n+1}^{(4)}}{\left(I_{n}^{(4)}\right)^{2}}= \begin{cases}\frac{2 n(2 n+1)}{4 b^{2}} & \text { (Gauss) } \\ \frac{2 n(2 n+1)(2 n+a)(2 n+a-1)}{b^{4}} & \text { (Laguerre) } \\ \frac{Q}{Q_{6}^{ \pm}} & \text {(Jacobi) }\end{cases}
$$

satisfy the following functional dependence:

$$
b_{n}^{(4)}(n, a, b)=b_{n}^{(1)}\left(-2 n,-\frac{a}{2},-\frac{b}{2}\right) .
$$

In the expressions above, $Q$ (already appearing in (0.3.1)), and a new expression $Q_{6}^{ \pm}$are expressible in terms of the variables $q, r, s$ introduced in (0.3.1):

$$
Q:=\left\{\begin{array}{cc}
48(n-1) n(2 a+n)(2 a+n+1)(2 b+n)(2 b+n+1) & \\
(2 b+2 a+n+1)(2 b+2 a+n+2), & \text { for }(\beta=1) \\
96 n(2 n+1)(a+2 n-1)(a+2 n)(b+2 n-1) & \\
(b+2 n)(b+a+2 n-2)(b+a+2 n-1), & \text { for }(\beta=4) \\
& =\frac{3}{16}\left(\left(s^{2}-q r+q^{2}\right)^{2}-4\left(r s^{2}-4 q s^{2}-4 s^{2}+q^{2} r\right)\right)
\end{array}\right.
$$

and 11

$$
\begin{aligned}
& Q_{6}^{ \pm}=\left\{\begin{array}{cc}
=48(b+a+n)(b+a+n+1)^{2}(b+a+n+2)(2 b+2 a+2 n-1) \\
(2 b+2 a+2 n+1)^{2}(2 b+2 a+2 n+3), & \text { for } \beta=1 \\
=3(b+a+4 n-4)(b+a+4 n-3)(b+a+4 n-2)^{2} & \\
(b+a+4 n-1)^{2}(b+a+4 n)(b+a+4 n+1), & \text { for } \beta=4
\end{array}\right. \\
& =3 q(q+1)(q-3)(q+4 \pm 4 \sqrt{q+1}) \quad\left\{\begin{array}{l}
+ \text { for } \beta=1 \\
- \text { for } \beta=4
\end{array} .\right.
\end{aligned}
$$

Proof. For instance, in the Jacobi case, one computes

$$
\begin{aligned}
\frac{I_{n+2}^{(1)}}{I_{n}^{(1)}=} & 2^{2 n+2 a+2 b+3} \frac{\Gamma\left(\frac{n+3}{2}+a+b\right) \Gamma\left(\frac{n+4}{2}+a+b\right) \Gamma\left(\frac{n+2}{2}+a\right) \Gamma\left(\frac{n+2}{2}+b\right)}{\Gamma\left(n+a+b+\frac{3}{2}\right) \Gamma(n+a+b+2)} \\
& \cdot \frac{\Gamma\left(\frac{n+3}{2}+a\right) \Gamma\left(\frac{n+3}{2}+b\right) \Gamma\left(\frac{n+3}{2}\right) \Gamma\left(\frac{n+4}{2}\right)}{\Gamma\left(n+a+b+\frac{5}{2}\right) \Gamma(n+a+b+3)} \\
\frac{I_{n+1}^{(4)}}{I_{n}^{(4)}}= & 2^{4 n+a+b} \frac{\Gamma(2 n+a+b) \Gamma(2 n+a+1) \Gamma(2 n+b+1) \Gamma(2 n+3)}{\Gamma(4 n+a+b) \Gamma(4 n+a+b+2)}
\end{aligned}
$$

\footnotetext{
${ }^{11} \sqrt{q+1}=2 n+2 b+2 a+1$ for $\beta=1$ and $\sqrt{q+1}=4 n+b+a-1$ for $\beta=4$
} 
and so,

$$
\begin{aligned}
& \left.\frac{(n !)^{2}}{(n-2) !(n+2) !} \frac{I_{n-2}^{(\beta)} I_{n+2}^{(\beta)}}{\left(I_{n}^{(\beta)}\right)^{2}}\right|_{\beta=1}=\left.\frac{Q}{Q_{6}^{ \pm}}\right|_{\beta=1} \\
& \left.\frac{(n !)^{2}}{(n-1) !(n+1) !} \frac{I_{n-1}^{(\beta)} I_{n+1}^{(\beta)}}{\left(I_{n}^{(\beta)}\right)^{2}}\right|_{\beta=4}=\left.\frac{Q}{Q_{6}^{ \pm}}\right|_{\beta=4} .
\end{aligned}
$$

\section{Proof of Theorems $0.1,0.2,0.3$}

From Theorems 2.1 and 2.2, the integrals $I_{n}(t, c)$, depending on $\beta=$ $2,1,4$, on $t=\left(t_{1}, t_{2}, \ldots\right)$ and on the boundary points $c=\left(c_{1}, \ldots, c_{2 r}\right)$ of $E$, relate to $\tau$-functions, as follows:

$$
\begin{aligned}
& I_{n}(t, c)=\int_{E^{n}}\left|\Delta_{n}(z)\right|^{\beta} \prod_{k=1}^{n}\left(e^{\sum_{1}^{\infty} t_{i} z_{k}^{i}} \rho\left(z_{k}\right) d z_{k}\right) \\
& =\left\{\begin{array}{lll}
n ! \tau_{n}(t, c), & n \text { arbitrary, } & \beta=2 \\
n ! \tau_{n}(t, c), & n \text { even, } & \beta=1 \\
n ! \tau_{2 n}(t / 2, c), & n \text { arbitrary, } & \beta=4 .
\end{array}\right.
\end{aligned}
$$

$I_{n}(t)$ refers to the integral (4.0.1) over the full range. It also follows that $\tau_{n}(t, c)$ satisfies the KP-like equation 12

$$
12 \frac{\tau_{n-2}(t, c) \tau_{n+2}(t, c)}{\tau_{n}(t, c)^{2}} \delta_{1,4}^{\beta}=(\mathrm{KP})_{t} \log \tau_{n}(t, c), \quad\left\{\begin{array}{l}
n \text { arbitrary for } \beta=2 \\
n \text { even for } \beta=1,4
\end{array}\right.
$$

where

$$
(\mathrm{KP})_{t} F:=\left(\left(\frac{\partial}{\partial t_{1}}\right)^{4}+3\left(\frac{\partial}{\partial t_{2}}\right)^{2}-4 \frac{\partial^{2}}{\partial t_{1} \partial t_{3}}\right) F+6\left(\frac{\partial^{2}}{\partial t_{1}^{2}} F\right)^{2}
$$

4.1. $\beta=2$,1. Evaluating the left-hand side of $(4.0 .2)$ (for $\beta=1$ ) yields, taking into account $P_{n}:=P_{n}(E)=I_{n}(0, c) / I_{n}(0)$ :

$$
\begin{aligned}
\left.12 \frac{\tau_{n-2}(t, c) \tau_{n+2}(t, c)}{\tau_{n}(t, c)^{2}}\right|_{t=0} & =\left.12 \frac{(n !)^{2}}{(n-2) !(n+2) !} \frac{I_{n-2}(t, c) I_{n+2}(t, c)}{I_{n}(t, c)^{2}}\right|_{t=0} \\
& =12 \frac{n(n-1)}{(n+1)(n+2)} \frac{I_{n-2}(0) I_{n+2}(0)}{I_{n}(0)^{2}} \frac{P_{n-2} P_{n+2}}{P_{n}^{2}} \\
& =12 b_{n}^{(1)} \frac{P_{n-2}(E) P_{n+2}(E)}{P_{n}^{2}(E)}
\end{aligned}
$$

\footnotetext{
${ }^{12}$ Remember $\delta_{1,4}^{\beta}=1$ for $\beta=1,4$, and $=0$ for $\beta=2$.
} 
with $b_{n}^{(1)}$ given by Lemma 3.1. Concerning the right-hand side of (4.0.2), it follows from Section 2.1 that $F_{n}(t ; c)=\log I_{n}(t ; c)$, as in (4.0.1), satisfies Virasoro constraints, corresponding precisely to the situation of Sections 3.1 and 3.2 for Gauss, Laguerre and Jacobi. As explained in (3.1.4), (3.2.1) and (3.2.2), we express

$$
\left.\frac{\partial^{4} F}{\partial t_{1}^{4}}\right|_{t=0},\left.\frac{\partial^{2} F}{\partial t_{2}^{2}}\right|_{t=0},\left.\frac{\partial^{2} F}{\partial t_{1} \partial t_{3}}\right|_{t=0},\left.\frac{\partial^{2} F}{\partial t_{1}^{2}}\right|_{t=0}, \quad F=\log I_{n}(t, c),
$$

in terms of $\mathcal{D}_{k}$ and $\mathcal{B}_{k}$, which when substituted in the right-hand side of (4.0.2), i.e., in the KP-expressions, leads to (upon comparing the expressions (1.3.4) and (1.3.8) with (3.1.1) for Gauss and Laguerre and using (3.2.1) directly for Jacobi):

- Gauss with $\left\{\begin{array}{l}\gamma_{1,-1}=-\frac{1}{2}, \gamma_{1,0}=\gamma_{1}=0, \delta_{1}=-\frac{n}{2} \\ \gamma_{2,-1}=0, \gamma_{2,0}=-1 / 2, \gamma_{2,1}=0, \gamma_{2}=-\frac{n}{4} \sigma_{1}, \delta_{2}=0 \\ \gamma_{3,-1}=-\frac{1}{4} \sigma_{1}, \gamma_{3,0}=0, \gamma_{3,1}=-\frac{1}{2}, \gamma_{3,2}=\gamma_{3}=0, \delta_{3}=-\frac{n}{4} \sigma_{1} .\end{array}\right.$

$\left.(\mathrm{KP})_{t} \log \tau_{n}(t, c)\right|_{t=0}$

$$
\begin{gathered}
=\left(\mathcal{D}_{1}^{4}+6 n \mathcal{D}_{1}^{2}+3 \mathcal{D}_{2}^{2}-3 \mathcal{D}_{2}-4 \mathcal{D}_{1} \mathcal{D}_{3}\right) F+6\left(\mathcal{D}_{1}^{2} F\right)^{2}+\frac{3}{4}(2-\beta) n(n-1) \\
=\frac{1}{16}\left(\left(\mathcal{B}_{-1}^{4}+8(n+(2-\beta)(n-1)) \mathcal{B}_{-1}^{2}+12 \mathcal{B}_{0}^{2}+24 \mathcal{B}_{0}-16 \mathcal{B}_{-1} \mathcal{B}_{1}\right) F\right. \\
\left.+6\left(\mathcal{B}_{-1}^{2} F\right)^{2}+12(2-\beta) n(n-1)\right)
\end{gathered}
$$

- Laguerre with $\left\{\begin{array}{l}\gamma_{1,-1}=0, \gamma_{1,0}=-1, \gamma_{1}=-\frac{n}{2}\left(\sigma_{1}+a\right), \\ \gamma_{2,-1}=0, \gamma_{2,0}=-\sigma_{1}, \gamma_{2,1}=-1, \gamma_{2}=-\frac{n}{2} \sigma_{1}\left(\sigma_{1}+a\right), \\ \gamma_{3,-1}=0, \gamma_{3,0}=-\sigma_{1} \sigma_{2}, \gamma_{3,1}=-\sigma_{2}, \\ \gamma_{3,2}=-1, \gamma_{3}=-\frac{n}{2} \sigma_{1} \sigma_{2}\left(\sigma_{1}+a\right) .\end{array}\right.$

$$
\begin{aligned}
(\mathrm{KP})_{t} & \left.\log \tau_{n}(t, c)\right|_{t=0} \\
= & \left(\mathcal{D}_{1}^{4}-2(\beta-3) \mathcal{D}_{1}^{3}\right. \\
\quad & -\left(2 n(n-1)(\beta-2)(\beta-1)+(\beta-2)(4 a n+4 n+5)-4 n^{2}-4 a n-1\right) \mathcal{D}_{1}^{2} \\
\quad & -3(\beta-2)\left(\beta n^{2}-\beta n+2 a n+2 n+1\right) \mathcal{D}_{1} \\
& \left.+3 \mathcal{D}_{2}^{2}+6(\beta(n-1)+a+2) \mathcal{D}_{2}-6 \mathcal{D}_{3}-4 \mathcal{D}_{1} \mathcal{D}_{3}\right) F_{n}
\end{aligned}
$$




$$
\begin{aligned}
& -3(\beta-2)\left(\mathcal{D}_{1} F_{n}\right)^{2}+6\left(\mathcal{D}_{1}^{2} \log \tau_{N}\right)^{2}-4(\beta-3)\left(\mathcal{D}_{1} F_{n}\right)\left(\mathcal{D}_{1}^{2} F_{n}\right) \\
& -\frac{3}{4}(\beta-2) n(n-1)(\beta n-2 \beta+2 a+2)(\beta n-\beta+2 a+2) \\
= & \left(\mathcal{B}_{-1}^{4}+2(\beta-3) \mathcal{B}_{-1}^{3}\right. \\
& -\left((\beta-2)\left(3(\beta-1)(n-1)^{2}+3 n^{2}+6 a n-4 a+2\right)+\left(a^{2}-1\right)\right) \mathcal{B}_{-1}^{2} \\
& +3(\beta-2)\left((\beta-1)(n-1)^{2}+n^{2}+2 a n-a\right) \mathcal{B}_{-1}-4 \mathcal{B}_{1} \mathcal{B}_{-1}-2 \mathcal{B}_{1} \\
& \left.+2(\beta n+a) \mathcal{B}_{0} \mathcal{B}_{-1}+3 \mathcal{B}_{0}^{2}-(\beta n+a) \mathcal{B}_{0}\right) F \\
& +6\left(\mathcal{B}_{-1}^{2} F\right)^{2}+4(\beta-3)\left(\mathcal{B}_{-1} F\right)\left(\mathcal{B}_{-1}^{2} F\right)+3(2-\beta)\left(\mathcal{B}_{-1} F\right)^{2} \\
& -\frac{3}{4}(\beta-2) n(n-1)(\beta n-2 \beta+2 a+2)(\beta n-\beta+2 a+2) .
\end{aligned}
$$

- Jacobi13

$$
\text { for } \beta=2 \text {, }
$$

$$
\begin{aligned}
\left.\frac{1}{8} q\left(q^{2}-4\right)(\mathrm{KP})_{t} \log \tau_{n}(t, c)\right|_{t=0} & \left(2 \mathcal{B}_{-1}^{4}+(q-r+4) \mathcal{B}_{-1}^{2}-\left(4 \mathcal{B}_{-1} F-s\right) \mathcal{B}_{-1}+3 q \mathcal{B}_{0}^{2}-2 q \mathcal{B}_{0}+8 \mathcal{B}_{0} \mathcal{B}_{-1}^{2}\right. \\
= & \left.4(q-1) \mathcal{B}_{1} \mathcal{B}_{-1}+\left(4 \mathcal{B}_{-1} F-s\right) \mathcal{B}_{1}+2\left(4 \mathcal{B}_{-1} F-s\right) \mathcal{B}_{0} \mathcal{B}_{-1}+2 q \mathcal{B}_{2}\right) F \\
& +4 \mathcal{B}_{-1}^{2} F\left(2 \mathcal{B}_{0} F+3 \mathcal{B}_{-1}^{2} F\right)
\end{aligned}
$$

for $\beta=1$,

$$
\begin{aligned}
\left.Q_{6}^{ \pm}(\mathrm{KP})_{t} \log \tau_{n}(t, c)\right|_{t=0} \\
=\quad(q+1)\left(4 q \mathcal{B}_{-1}^{4}+12\left(4 \mathcal{B}_{-1} F-s\right) \mathcal{B}_{-1}^{3}+2(q+12)\left(4 \mathcal{B}_{-1} F-s\right) \mathcal{B}_{0} \mathcal{B}_{-1}\right. \\
\left.\quad+3 q^{2} \mathcal{B}_{0}^{2}-4(q-4) q \mathcal{B}_{1} \mathcal{B}_{-1}+q\left(4 \mathcal{B}_{-1} F-s\right) \mathcal{B}_{1}+20 q \mathcal{B}_{0} \mathcal{B}_{-1}^{2}+2 q^{2} \mathcal{B}_{2}\right) F \\
\quad+\left(Q_{2} \mathcal{B}_{-1}^{2}-s Q_{1} \mathcal{B}_{-1}+Q_{3} \mathcal{B}_{0}\right) F+48\left(\mathcal{B}_{-1} F\right)^{4} \\
\quad-48 s\left(\mathcal{B}_{-1} F\right)^{3}+2 Q_{4}\left(\mathcal{B}_{-1} F\right)^{2} \\
\quad+12 q^{2}\left(\mathcal{B}_{0} F\right)^{2}+16 q(2 q-1) \mathcal{B}_{-1}^{2} F \mathcal{B}_{0} F+24(q-1) q\left(\mathcal{B}_{-1}^{2} F\right)^{2} \\
\quad+24\left(2 \mathcal{B}_{-1} F-s\right) \mathcal{B}_{-1} F\left((q+2) \mathcal{B}_{0} F+(q+3) \mathcal{B}_{-1}^{2} F\right)+Q
\end{aligned}
$$

\footnotetext{
${ }^{13}$ In the Jacobi $\beta=2$ case, we have $b_{0}=a-b, b_{1}=a+b$; thus $r=2\left(b_{0}^{2}+b_{1}^{2}\right), q_{n}=2(2 n+a+b)^{2}$ and $q\left(q^{2}-4\right)=16(2 n+\gamma+\delta)^{2}(2 n+\gamma+\delta-1)(2 n+\gamma+\delta+1)$.
} 
where the $Q_{1}, Q_{2}, Q_{3}, Q_{4}, Q$ are given by (0.3.1) and where the auxiliary $Q_{6}^{ \pm}$ happens to be exactly the one of Lemma 3.1. This establishes Theorems 0.1, 0.2 and 0.3 for $\beta=2,1$, at least when $b=1$ in the exponent of the Gaussian and Laguerre ensembles, upon noting that $\mathcal{B}_{k}^{j} \log P_{n}(E)=\mathcal{B}_{k}^{j} \log I_{n}(0, c) / I_{n}(0)=$ $\mathcal{B}_{k}^{j} \log \tau_{n}(0, c)$.

Finally, a simple argument captures the case $b \neq 1$. Indeed, setting $\alpha E:=$ $\bigcup_{1}^{2 r}\left[\begin{array}{ll}\alpha & c_{2 i-1}, \alpha c \\ c_{2 i}\end{array}\right] \subset F$, for $\alpha>0$, the elementary identities

$$
\begin{aligned}
& I_{n}(t, c)=\int_{E^{n}}\left|\Delta_{n}(z)\right|^{\beta} \prod_{k=1}^{n} e^{-b z_{k}^{2}} d z_{k}=C \int_{(\sqrt{b} E)^{n}}\left|\Delta_{n}(z)\right|^{\beta} \prod_{k=1}^{n} e^{-z_{k}^{2}} d z_{k} \\
& I_{n}(t, c)=\int_{E^{n}}\left|\Delta_{n}(z)\right|^{\beta} \prod_{k=1}^{n} z_{k}^{a} e^{-b z_{k}} d z_{k}=C \int_{(b E)^{n}}\left|\Delta_{n}(z)\right|^{\beta} \prod_{k=1}^{n} z_{k}^{a} e^{-z_{k}} d z_{k},
\end{aligned}
$$

where $C(a, b, n, \beta)$ is a constant independent of $E$, lead to the same Virasoro constraints as in Examples 1 and $2(\S 1.3)$, but with the following mapping for the differential operators

$$
\begin{aligned}
\left(\mathcal{B}_{-1}, \mathcal{B}_{0}, \mathcal{B}_{1}\right) & \rightarrow\left(\frac{\mathcal{B}_{-1}}{\sqrt{b}}, \mathcal{B}_{0}, \mathcal{B}_{1} \sqrt{b}\right) \quad \text { (Gauss) } \\
& \rightarrow\left(\mathcal{B}_{-1}, b \mathcal{B}_{0}, b^{2} \mathcal{B}_{1}\right) \quad \text { (Laguerre) }
\end{aligned}
$$

Therefore, the equations (0.1.2) and (0.2.2) for the probabilities (0.1.1) and (0.2.1) are obtained by making the substitutions (4.1.1) and (4.1.2) in the PDEs (0.1.2) $\left.\right|_{b=1}$ and (0.2.2) $\left.\right|_{b=1}$; this process yields the precise equations (0.1.2) and (0.2.2), with $b \neq 1$. This ends the proof of Theorems 0.1, 0.2 and 0.3 for the cases $\beta=1,2$.

4.2. $\beta=4$, using duality. From (4.0.1), the integral for $\beta=4$ is expressible in terms of a $\tau$-function, in which $t$ is replaced by $t / 2$. Hence (4.0.2) becomes:

$$
12 \frac{\tau_{2 n-2}(t / 2, c) \tau_{2 n+2}(t / 2, c)}{\tau_{2 n}(t / 2, c)^{2}}=(\mathrm{KP})_{t / 2}\left(\log \tau_{2 n}\right)(t / 2, c) .
$$

So, the left-hand side of (4.2.1) equals $\left(P_{n}:=P_{n}(E)=I_{n}(0, c) / I_{n}(0)\right)$

$$
\begin{aligned}
\left.12 \frac{\tau_{2 n-2}(t / 2, c) \tau_{2 n+2}(t / 2, c)}{\tau_{2 n}(t / 2, c)^{2}}\right|_{t=0} & =\left.12 \frac{(n !)^{2}}{(n-1) !(n+1) !} \frac{I_{n-1}(t, c) I_{n+1}(t, c)}{I_{n}(t, c)^{2}}\right|_{t=0} \\
& =12 \frac{n}{(n+1)} \frac{I_{n-1}(0) I_{n+1}(0)}{I_{n}(0)^{2}} \frac{P_{n-1} P_{n+1}}{P_{n}^{2}} \\
& =12 b_{n}^{(4)} \frac{P_{n-1}(E) P_{n+1}(E)}{P_{n}^{2}(E)}
\end{aligned}
$$

where $b_{n}^{(4)}=b_{n}^{(4)}(n, a, b)$ is given by Lemma 3.1 and satisfies

$$
b_{n}^{(4)}(n, a, b)=b_{n}^{(1)}\left(-2 n,-\frac{a}{2},-\frac{b}{2}\right) .
$$


Recall from Theorem $1.1(1.1 .4)$ that $I_{n}^{(\beta)}\left(t, c ; a_{i}, b_{i}\right)$ and $I_{n}^{(4 / \beta)}\left(t, c ; a_{i}, b_{i}\right)$ (where we indicate the explicit dependence on the coefficients $a_{i}$ and $b_{i}$ of $\rho^{\prime} / \rho$ ) satisfy the same equations, with altered parameters:

$$
\begin{gathered}
\left(\mathcal{B}_{k}-\mathcal{V}_{k, n}^{(\beta)}\left(t ; n, a_{i}, b_{i}\right)\right) I_{n}^{(\beta)}\left(t, c ; a_{i}, b_{i}\right)=0 \\
\left(\mathcal{B}_{k}-\mathcal{V}_{k, n}^{(\beta)}\left(-\frac{\beta}{2} t ;-\frac{2}{\beta} n, a_{i},-\frac{\beta}{2} b_{i}\right)\right) I_{n}^{(4 / \beta)}\left(t, c ; a_{i}, b_{i}\right)=0 .
\end{gathered}
$$

Setting $\beta=1$ in the equations above, extracting $t$-partials in terms of $\mathcal{B}_{k}$ 's, and using the procedure explained in this section, we have that

$$
\begin{aligned}
\left.(\mathrm{KP})_{t}\left(\log I_{n}^{(1)}\left(t, c ; a_{i}, b_{i}\right)\right)\right|_{t=0} & =R\left(\mathcal{B} ; n, a_{i}, b_{i}\right) \log I_{n}^{(1)}\left(0, c ; a_{i}, b_{i}\right) \\
& =R\left(\mathcal{B} ; n, a_{i}, b_{i}\right) \log P_{n}^{(1)}(E), \\
\left.(\mathrm{KP})_{t / 2}\left(\log I_{n}^{(4)}\left(t, c ; a_{i}, b_{i}\right)\right)\right|_{t=0} & =\left.(\mathrm{KP})_{-t / 2}\left(\log I_{n}^{(4)}\left(t, c ; a_{i}, b_{i}\right)\right)\right|_{t=0} \\
& =R\left(\mathcal{B} ;-2 n, a_{i},-b_{i} / 2\right) \log I_{n}^{(4)}\left(0, c ; a_{i}, b_{i}\right) \\
& =R\left(\mathcal{B} ;-2 n, a_{i},-b_{i} / 2\right) \log P_{n}^{(4)}(E),
\end{aligned}
$$

where $R\left(\mathcal{B} ; a_{i}, b_{i}, n\right)$ denotes the right-hand side of the equations (0.1.2), (0.2.2) and (0.3.4) for $\beta=1$. The coefficients $a_{i}$ and $b_{i}$ of the rational function $-\rho^{\prime} / \rho$ are as follows: the $a_{i}$ and $b_{i}$ all vanish, except for

$$
\begin{array}{llllll}
\text { Hermite } & a_{0}=1 & a_{1}=0 & a_{2}=0 & b_{0}=0 & b_{1}=2 b \\
\text { Laguerre } & a_{0}=0 & a_{1}=1 & a_{2}=0 & b_{0}=-a & b_{1}=b \\
\text { Jacobi } & a_{0}=1 & a_{1}=0 & a_{2}=-1 & b_{0}=a-b & b_{1}=a+b ;
\end{array}
$$

thus the map

$$
\left(n, a_{i}, b_{i}\right) \longrightarrow\left(-2 n, a_{i},-b_{i} / 2\right)
$$

translates into the map

$$
(n, a, b) \longrightarrow(-2 n,-a / 2,-b / 2),
$$

which shows that the PDEs (0.1.2), (0.2.2) and (0.3.4) for the case $\beta=4$ are obtained by means of the map (4.2.2) from the same PDEs for $\beta=1$. But according to (0.0.5), this is the precise way the coefficients $q, s, Q_{-1}, Q_{0}, Q_{1}, Q_{2}$, $Q_{3}, Q_{4}, Q$, evaluated at $\beta=4$, are obtained from the same coefficients at $\beta=1$. This ends the proof of Theorem 0.3.

4.3. Reduction to Chazy and Painlevé equations $(\beta=2)$. Setting $E=$ $[-\infty, x], E=[0, x], E=[-1, x]$ in the PDEs $(0.1 .2),(0.2 .2)$ and (0.3.4) respectively, leads to the equations $(0.4 .1),(0.4 .2)$ and (0.4.3) respectively, as announced in Section 0.4. Furthermore setting $\beta=2$, the inductive terms on the left-hand side of (0.4.1) and (0.4.2) vanish and one obtains the ODEs: 
- Gauss: $P_{n}\left(\max _{i} \lambda_{i} \leq x\right)=\exp \left(-\int_{x}^{\infty} f(u) d u\right)$, where $f$ satisfies:

$$
f^{\prime \prime \prime}+6 f^{\prime 2}+4 b\left(2 n-b x^{2}\right) f^{\prime}+4 b^{2} x f=0 .
$$

- Laguerre: $P_{n}\left(\max _{i} \lambda_{i} \leq x\right)=\exp \left(-\int_{x}^{\infty} \frac{f(u)}{u} d u\right)$, where $f$ satisfies $x^{2} f^{\prime \prime \prime}+x f^{\prime \prime}+6 x f^{\prime 2}-4 f f^{\prime}-\left((a-b x)^{2}-4 n b x\right) f^{\prime}-b(2 n+a-b x) f=0$.

- Jacobi: $P_{n}\left(\max _{i} \lambda_{i} \leq x\right)=\exp \left(-\int_{x}^{1} \frac{f(u)}{1-u^{2}} d u\right)$, where $f$ satisfies:

$$
\begin{aligned}
2\left(x^{2}-1\right)^{2} f^{\prime \prime \prime}+4\left(x^{2}-1\right)\left(x f^{\prime \prime}-3 f^{\prime 2}\right)+ & \left(16 x f-q_{n}\left(x^{2}-1\right)-2 s x-r\right) f^{\prime} \\
& -f\left(4 f-q_{n} x-s\right)=0
\end{aligned}
$$

where $r, s, q_{n}$ are defined in (0.3.1).

These three equations are of the form

$$
f^{\prime \prime \prime}+\frac{P^{\prime}}{P} f^{\prime \prime}+\frac{6}{P} f^{\prime 2}-\frac{4 P^{\prime}}{P^{2}} f f^{\prime}+\frac{P^{\prime \prime}}{P^{2}} f^{2}+\frac{4 Q}{P^{2}} f^{\prime}-\frac{2 Q^{\prime}}{P^{2}} f+\frac{2 R}{P^{2}}=0,
$$

with the following coefficients $P, Q, R$ :

$$
\begin{array}{llll}
\text { Gauss } & P(x)=1 & 4 Q(x)=-4 b^{2} x^{2}+8 b n & R=0 \\
\text { Laguerre } & P(x)=x & 4 Q(x)=-(b x-a)^{2}+4 b n x & R=0 \\
\text { Jacobi } & P(x)=1-x^{2} & 4 Q(x)=-\frac{1}{2}\left(q_{n}\left(x^{2}-1\right)+2 s x+r\right) & R=0 .
\end{array}
$$

The general Chazy class of differential equations are equations of the form $f^{\prime \prime \prime}=F\left(z, f, f^{\prime}, f^{\prime \prime}\right)$, where $F$ is rational in $f, f^{\prime}, f^{\prime \prime}$ and locally analytic in z, subjected to the requirement that the general solution be free of movable branch points; the latter is a branch point whose location depends on the integration constants. In his classification, Chazy found thirteen cases, the first of which is given by (4.3.1), with arbitrary polynomials $P(z), Q(z), R(z)$ of degree $3,2,1$ respectively.

Cosgrove $([11],[12]),($ A.3), shows this third-order equation has a first integral, which is second-order in $f$ and quadratic in $f^{\prime \prime}$,

$$
\begin{array}{r}
f^{\prime \prime 2}+\frac{4}{P^{2}}\left(\left(P f^{\prime 2}+Q f^{\prime}+R\right) f^{\prime}-\left(P^{\prime} f^{\prime 2}+Q^{\prime} f^{\prime}+R^{\prime}\right) f\right. \\
\left.+\frac{1}{2}\left(P^{\prime \prime} f^{\prime}+Q^{\prime \prime}\right) f^{2}-\frac{1}{6} P^{\prime \prime \prime} f^{3}+c\right)=0
\end{array}
$$


with an integration constant $c$. In the three cases, discussed above, $c=0$. Notice equations of the general form

$$
f^{\prime \prime 2}=G\left(x, f, f^{\prime}\right)
$$

are invariant under the map

$$
x \mapsto \frac{a_{1} z+a_{2}}{a_{3} z+a_{4}} \text { and } f \mapsto \frac{a_{5} f+a_{6} z+a_{7}}{a_{3} z+a_{4}} .
$$

Using this map, the polynomial $P(z)$ can be normalized to

$$
P(z)=z(z-1), z, \text { or } 1 .
$$

Equation (4.3.2) is a master Painlevé equation, containing the six Painlevé equations. If $f(x)$ satisfies the first three equations above, then the new function $g(z)$, defined below,

$$
\begin{array}{ll}
\text { Gauss } & g(z)=b^{-1 / 2} f\left(z b^{-1 / 2}\right)+\frac{2}{3} n z \\
\text { Laguerre } & g(z)=f(z)+\frac{b}{4}(2 n+a) z+\frac{a^{2}}{4} \\
\text { Jacobi } & g(z):=-\left.\frac{1}{2} f(x)\right|_{x=2 z-1}-\frac{q}{8} z+\frac{q+s}{16}
\end{array}
$$

satisfies the following canonical equations of Cosgrove and Scoufis ([11], [12]):

- $g^{\prime \prime 2}=-4 g^{\prime 3}+4\left(z g^{\prime}-g\right)^{2}+A_{1} g^{\prime}+A_{2}$,

(Painlevé IV)

- $\left(z g^{\prime \prime}\right)^{2}=\left(z g^{\prime}-g\right)\left(-4 g^{2}+A_{1}\left(z g^{\prime}-g\right)+A_{2}\right)+A_{3} g^{\prime}+A_{4}$,

(Painlevé V)

- $\left(z(z-1) g^{\prime \prime}\right)^{2}=\left(z g^{\prime}-g\right)\left(4 g^{\prime 2}-4 g^{\prime}\left(z g^{\prime}-g\right)+A_{2}\right)+A_{1} g^{2}+A_{3} g^{\prime}+A_{4}$

(Painlevé VI)

with respective coefficients

- $A_{1}=3\left(\frac{4 n}{3}\right)^{2}, A_{2}=-\left(\frac{4 n}{3}\right)^{3}$,

- $A_{1}=b^{2}, A_{2}=b^{2}\left(\left(n+\frac{a}{2}\right)^{2}+\frac{a^{2}}{2}\right), A_{3}=-a^{2} b\left(n+\frac{a}{2}\right), A_{4}=\frac{(a b)^{2}}{2}$

$$
\cdot\left(\left(n+\frac{a}{2}\right)^{2}+\frac{a^{2}}{8}\right) \text {, }
$$

- $A_{1}=\frac{2 q+r}{8}, A_{2}=\frac{q s}{16}, A_{3}=\frac{(q-s)^{2}+2 q r}{64}, A_{4}=\frac{q}{512}\left(2 s^{2}+q r\right)$.

Each of the equations above can be transformed into the standard Painlevé equations. 


\section{Appendix. Self-similarity proof of the Virasoro constraints (Theorem 1.1)}

Given the data (0.0.1) to (0.0.3), namely $\rho=e^{-V}$ and $-\rho^{\prime} / \rho=V^{\prime}=$ $g / f=\sum_{0}^{\infty} b_{i} z^{i} / \sum_{0}^{\infty} a_{i} z^{i}$ and $E=\bigcup_{1}^{r}\left[c_{2 i-1}, c_{2 i}\right] \subseteq F \subseteq \mathbb{R}$, we show that the multiple integral

$$
I_{n}(t, c ; \beta):=\int_{E^{n}}\left|\Delta_{n}(x)\right|^{\beta} \prod_{k=1}^{n}\left(e^{\sum_{1}^{\infty} t_{i} x_{k}^{i}} \rho\left(x_{k}\right) d x_{k}\right), \text { for } n>0
$$

satisfies the Virasoro constraints of Theorem 1.1, using a (much less conceptual!) self-similarity argument. Setting

$$
d I_{n}(x):=\left|\Delta_{n}(x)\right|^{\beta} \prod_{k=1}^{n}\left(e^{\sum_{1}^{\infty} t_{i} x_{k}^{i}} \rho\left(x_{k}\right) d x_{k}\right),
$$

we state the following lemma:

LEMMA 5.1. The following variational formula holds:

$$
\left.\frac{d}{d \varepsilon} d I_{n}\left(x_{i} \mapsto x_{i}+\varepsilon f\left(x_{i}\right) x_{i}^{k+1}\right)\right|_{\varepsilon=0}=\sum_{\ell=0}^{\infty}\left(a_{\ell}{ }^{\beta} \mathbb{J}_{k+\ell, n}^{(2)}-b_{\ell}{ }^{\beta} \mathbb{J}_{k+\ell+1, n}^{(1)}\right) d I_{n} .
$$

Proof. Upon setting

$$
\begin{aligned}
E(x, t) & :=\prod_{1}^{n} e^{\sum_{i=1}^{\infty} t_{i} x_{k}^{i}} \rho\left(x_{k}\right) \\
& =\prod_{1}^{n} e^{-V\left(x_{k}, t\right)}, \text { where } V(x, t):=V(x)-\sum_{1}^{\infty} t_{i} x^{i},
\end{aligned}
$$

the following two relations hold:

$$
\begin{aligned}
\left(\frac{1}{2} \sum_{\substack{i+j=k \\
i, j>0}} \frac{\partial^{2}}{\partial t_{i} \partial t_{j}}-\frac{n}{2} \delta_{k, 0}\right) E & =\left(\sum_{\substack{1 \leq a<\beta \leq n \\
i, j>0 \\
i+j=k}} x_{\alpha}^{i} x_{\beta}^{j}+\frac{k-1}{2} \sum_{1 \leq \alpha \leq n} x_{\alpha}^{k}\right) E, \\
\left(\frac{\partial}{\partial t_{k}}+n \delta_{k, 0}\right) E & =\left(\sum_{1 \leq \alpha \leq n} x_{\alpha}^{k}\right) E, \text { for all } k \geq 0 .
\end{aligned}
$$

So, the point now is to compute the $\varepsilon$-derivative

$$
\left.\frac{d}{d \varepsilon}\left(\left|\Delta_{n}(x)\right|^{\beta} e^{\sum_{k=1}^{n}\left(-V\left(x_{k}\right)+\sum_{i=1}^{\infty} t_{i} x_{k}^{i}\right)} d x_{1} \ldots d x_{n}\right)_{x_{i} \mapsto x_{i}+\varepsilon f\left(x_{i}\right) x_{i}^{k+1}}\right|_{\varepsilon=0},
$$

which consists of three contributions: 
Contribution 1:

$$
\begin{aligned}
& \left.\frac{\partial}{\partial \varepsilon}\left|\Delta\left(x+\varepsilon f(x) x^{k+1}\right)\right|^{\beta}\right|_{\varepsilon=0} \\
& =\left.\beta|\Delta(x)|^{\beta} \sum_{1 \leq \alpha<\gamma \leq n} \frac{\partial}{\partial \varepsilon} \log \left(\left|x_{\alpha}-x_{\gamma}+\varepsilon\left(f\left(x_{\alpha}\right) x_{\alpha}^{k+1}-f\left(x_{\gamma}\right) x_{\gamma}^{k+1}\right)\right|\right)\right|_{\varepsilon=0} \\
& =\beta|\Delta(x)|^{\beta} \sum_{1 \leq \alpha<\gamma \leq n} \frac{f\left(x_{\alpha}\right) x_{\alpha}^{k+1}-f\left(x_{\gamma}\right) x_{\gamma}^{k+1}}{x_{\alpha}-x_{\gamma}} \\
& =\beta|\Delta(x)|^{\beta} \sum_{\ell=0}^{\infty} a_{\ell} \sum_{1 \leq \alpha<\gamma \leq n} \frac{x_{\alpha}^{k+\ell+1}-x_{\gamma}^{k+\ell+1}}{x_{\alpha}-x_{\gamma}} \\
& =\beta|\Delta(x)|^{\beta} \sum_{\ell=0}^{\infty} a_{\ell}\left(\sum_{\substack{i+j=\ell+k \\
i, j>0 \\
1 \leq \alpha<\gamma \leq n}} x_{\alpha}^{i} x_{\gamma}^{j}+(n-1) \sum_{1 \leq \alpha \leq n} x_{\alpha}^{\ell+k}-\frac{n(n-1)}{2} \delta_{\ell+k, 0}\right) \\
& =\beta E^{-1}|\Delta(x)|^{\beta} \sum_{\ell=0}^{\infty} a_{\ell}\left(\frac{1}{2} \sum_{\substack{i+j=k+\ell \\
i, j>0}} \frac{\partial^{2}}{\partial t_{i} \partial t_{j}}-\frac{n}{2} \delta_{k+\ell, 0}\right. \\
& \left.+\left(n-\frac{k+\ell+1}{2}\right)\left(\frac{\partial}{\partial t_{k+\ell}}+n \delta_{k+\ell, 0}\right)-\frac{n(n-1)}{2} \delta_{k+\ell, 0}\right) E \\
& =\beta E^{-1}|\Delta(x)|^{\beta} \sum_{\ell=0}^{\infty} a_{\ell} \\
& \left(\frac{1}{2} \sum_{\substack{i+j=k+\ell \\
i, j>0}} \frac{\partial^{2}}{\partial t_{i} \partial t_{j}}+\left(n-\frac{k+\ell+1}{2}\right) \frac{\partial}{\partial t_{k+\ell}}+\frac{n(n-1)}{2} \delta_{k+\ell, 0}\right) E .
\end{aligned}
$$

Contribution 2:

$$
\begin{aligned}
\frac{\partial}{\partial \varepsilon} & \left.\prod_{1}^{n} d\left(x_{\alpha}+\varepsilon f\left(x_{\alpha}\right) x_{\alpha}^{k+1}\right)\right|_{\varepsilon=0} \\
& =\sum_{1}^{n}\left(f^{\prime}\left(x_{\alpha}\right) x_{\alpha}^{k+1}+(k+1) f\left(x_{\alpha}\right) x_{\alpha}^{k}\right) \prod_{1}^{n} d x_{i} \\
& =\sum_{\ell=0}^{\infty}(\ell+k+1) a_{\ell} \sum_{\alpha=1}^{n} x_{\alpha}^{k+\ell} \prod_{1}^{n} d x_{i} \\
& =E^{-1} \sum_{\ell=0}^{\infty}(\ell+k+1) a_{\ell}\left(\frac{\partial}{\partial t_{k+\ell}}+n \delta_{k+\ell, 0}\right) E \prod_{1}^{n} d x_{i},
\end{aligned}
$$




\section{Contribution 3:}

$$
\begin{aligned}
\frac{\partial}{\partial \varepsilon} \prod_{\alpha=1}^{n} \exp \left(-V\left(x_{\alpha}+\varepsilon f\left(x_{\alpha}\right) x^{k+1}\right)\right. & \left.\quad+\sum_{i=1}^{\infty} t_{i} \sum_{\alpha=1}^{n}\left(x_{\alpha}+\varepsilon f\left(x_{\alpha}\right) x_{\alpha}^{k+1}\right)^{i}\right)\left.\right|_{\varepsilon=0} \\
= & \left(-\sum_{\alpha=1}^{n} V^{\prime}\left(x_{\alpha}\right) f\left(x_{\alpha}\right) x_{\alpha}^{k+1}+\sum_{i=1}^{\infty} i t_{i} \sum_{\alpha=1}^{n} f\left(x_{\alpha}\right) x_{\alpha}^{i+k}\right) E \\
= & \left(-\sum_{\ell=0}^{\infty} b_{\ell} \sum_{\alpha=1}^{n} x_{\alpha}^{k+\ell+1}+\sum_{\ell \geq 0} a_{\ell} i t_{i} \sum_{\alpha=1}^{n} x_{\alpha}^{i+k+\ell}\right) E \\
= & \left(-\sum_{\ell=0}^{\infty} b_{\ell}\left(\frac{\partial}{\partial t_{k+\ell+1}}+n \delta_{k+\ell+1,0}\right)\right. \\
& \left.+\sum_{\ell=0}^{\infty} a_{\ell} \sum_{i=1}^{\infty} i t_{i}\left(\frac{\partial}{\partial t_{i+k+\ell}}+n \delta_{i+k+\ell, 0}\right)\right) E
\end{aligned}
$$

As mentioned, to conclude (5.0.2), we must add up the three contributions (5.0.6), (5.0.7) and (5.0.8), resulting in:

$$
\begin{aligned}
& \left.\frac{\partial}{\partial \varepsilon} d I_{n}\left(x_{i} \mapsto x_{i}+\varepsilon f\left(x_{i}\right) x_{i}^{k+1}\right)\right|_{\varepsilon=0} \\
& =\left(\sum _ { \ell = 0 } ^ { \infty } a _ { \ell } \left(\frac{\beta}{2} J_{k+\ell}^{(2)}+\left(n \beta+(\ell+k+1)\left(1-\frac{\beta}{2}\right)\right) J_{k+\ell}^{(1)}\right.\right. \\
& \left.\left.\quad+n\left((n-1) \frac{\beta}{2}+1\right) \delta_{k+\ell, 0}\right)-\sum_{\ell=0}^{\infty} b_{\ell}\left(J_{k+\ell+1}^{(1)}+n \delta_{k+\ell+1,0}\right)\right) d I_{n}(x) .
\end{aligned}
$$

where $J_{k}^{(i)}:={ }^{\beta} J_{k}^{(i)}$, as in (1.1.8). Thus we use (1.1.8) to end the proof of Lemma 5.1.

Proof of Theorem 1.1. The change of integration variable $x_{i} \mapsto x_{i}+$ $\varepsilon f\left(x_{i}\right) x_{i}^{k+1}$ in the integral (5.0.1) leaves the integral invariant, but it induces a change of limits of integration, given by the inverse of the map above; namely the $c_{i}$ 's in $E=\bigcup_{1}^{r}\left[c_{2 i-1}, c_{2 i}\right]$, get mapped as follows:

$$
c_{i} \mapsto c_{i}-\varepsilon f\left(c_{i}\right) c_{i}^{k+1}+O\left(\varepsilon^{2}\right) .
$$

Therefore, setting

$$
E^{\varepsilon}=\bigcup_{1}^{r}\left[c_{2 i-1}-\varepsilon f\left(c_{2 i-1}\right) c_{2 i-1}^{k+1}+O\left(\varepsilon^{2}\right), c_{2 i}-\varepsilon f\left(c_{2 i}\right) c_{2 i}^{k+1}+O\left(\varepsilon^{2}\right)\right],
$$


we find, using Lemma 5.1 and the fundamental theorem of calculus,

$$
\begin{aligned}
0 & =\frac{\partial}{\partial \varepsilon} \int_{\left(E^{\varepsilon}\right)^{2 n}}\left|\Delta_{2 n}\left(x+\varepsilon f(x) x^{k+1}\right)\right| \prod_{i=1}^{2 n} e^{-V\left(x_{i}+\varepsilon f\left(x_{i}\right) x_{i}^{k+1}, t\right)} d\left(x_{i}+\varepsilon f\left(x_{i}\right) x_{i}^{k+1}\right) \\
& =\left(-\sum_{i=1}^{2 r} c_{i}^{k+1} f\left(c_{i}\right) \frac{\partial}{\partial c_{i}}+\sum_{\ell=0}^{\infty}\left(a_{\ell}{ }^{\beta} \mathbb{J}_{k+\ell, n}^{(2)}-b_{\ell}{ }^{\beta} \mathbb{J}_{k+\ell+1, n}^{(1)}\right)\right) I_{n}(t, c, \beta) .
\end{aligned}
$$

This ends the alternative proof of Theorem 1.1.

Brandeis University, Waltham, MA 02454

E-mail address: adler@brandeis.edu

Université de Louvain, B1348 Louvain-LA-Neuve, Belgium

E-mail address: vanm@geom.ucl.ac.be and vanmoerbeke@brandeis.edu

\section{REFERENCES}

[1] M. Adler and P. van Moerbeke, Matrix integrals, Toda symmetries, Virasoro constraints and orthogonal polynomials, Duke Math. J. 80 (1995), 863-911.

[2] - The spectrum of coupled random matrices, Ann. of Math. 149 (1999), 921-976.

[3] Integrals over classical groups, random permutations, Toda and Toeplitz lattices, Comm. Pure Appl. Math. 54 (2001), 153-205 (math.CO9912143).

[4] The Pfaff lattice, matrix integrals and a map from Toda to Pfaff, Duke Math. J. (2001), to appear (solv-int/9912008).

[5] Generalized orthogonal polynomials, discrete KP and Riemann-Hilbert problems, Comm. Math. Phys. 207 (1999), 589-620.

[6] M. Adler, E. Horozov, and P. van Moerbeke, The Pfaff lattice and skew-orthogonal polynomials, Internat. Math. Res. Not. 11 (1999), 569-588.

[7] M. Adler, T. Shiota, and P. van Moerbeke, Random matrices, vertex operators and the Virasoro algebra, Phys. Lett. A 208 (1995), 67-78.

[8] , Pfaff $\tau$-functions, Math. Ann. (2001), to appear (bolv-int/9909010).

[9] K. Аомото, Jacobi polynomials associated with Selberg integrals, SIAM J. Math. Anal. 18 (1987), 545-549.

[10] H. Awata, Y. Matsuo, S. Odake, and J. Shiraishi, Collective field theory, CalogeroSutherland model and generalized matrix models, Phys. Lett. B 347 (1995), 49-55.

[11] C. M. Cosgrove, Chazy classes IX-XII of third-order differential equations, Stud. Appl. Math. 104 (2000), 171-228.

[12] C. M. Cosgrove and G. Scoufis, Painlevé classification of a class of differential equations of the second order and second degree, Stud. Appl. Math. 88 (1993), 25-87.

[13] L. Dickey, Soliton Equations and Hamiltonian Systems, World Scientific Publ. Co., River Edge, NJ (1991).

[14] L. Haine and J. P. Semengue, The Jacobi polynomial ensemble and the Painlevé VI equation, J. Math. Phys. 40 (1999), 2117-2134.

[15] G. Mahoux and M. L. Menta, A method of integration over matrix variables: IV, $J$. Physique I 1 (1991), 1093-1108.

[16] M. L. Мента, Random Matrices, 2nd ed. Academic Press, Boston, MA, 1991.

[17] $\longrightarrow$ A nonlinear differential equation and a Fredholm determinant, J. Physique I, (1992), 1721-1729.

[18] M. L. Мента and G. Maнoux, Level spacing functions and nonlinear differential equations, J. Physique I 3 (1993), 697-715. 
[19] G. Moore, Matrix models of 2D gravity and isomonodromic deformations, Progr. Theor. Phys. Suppl., No. 102 (1990), 255-285.

[20] C. A. Tracy and H. Widom, Fredholm determinants, differential equations and matrix models, Comm. Math. Phys. 163 (1994), 33-72.

[21] (1996), 727-754.

[22] K. Ueno and K. Takasaki, Toda lattice hierarchy, Adv. Stud. Pure Math. 4 (1984), 1-95.

[23] P. van Moerbeke, The spectrum of random matrices and integrable systems, Group 21, in Physical Applications and Mathematical Aspects of Geometry, Groups and Algebras, Vol. II, 835-852 (H.-D. Doebner, W. Scherer, and C. Schulte, eds.), World Scientific Publ. Co., Singapore, 1997.

[24] Integrable lattices: random matrices and random permutations, in Random Matrices and their Applications, MSRI publications 40 (2001), 321-406.

[25] E. P. Wigner, On the statistical distribution of the widths and spacings of nuclear resonance levels, Proc. Cambr. Philos. Soc. 47 (1951), 790-798.

(Received October 15, 1998) 